\title{
Roles of exosomes in cancer chemotherapy resistance, progression, metastasis and immunity, and their clinical applications (Review)
}

\author{
XIAOYAN WANG ${ }^{1,2^{*}}$, YUAN ZHOU ${ }^{3 *}$ and KAIYANG DING ${ }^{1}$ \\ ${ }^{1}$ Department of Hematology, The First Affiliated Hospital of USTC, Division of Life Sciences and Medicine, \\ University of Science and Technology of China, Hefei, Anhui $230001 ;{ }^{2}$ Gruduate School, Anhui Medical University, \\ Hefei, Anhui 230032; ${ }^{3}$ Gruduate School, Tianjin Medical University, Tianjin 300070, P.R. China
}

Received February 17, 2021; Accepted April 21, 2021

DOI: $10.3892 / \mathrm{ijo} .2021 .5224$

\begin{abstract}
Exosomes are a type of vesicle that are secreted by cells, with a diameter of 40-100 nm, and that appear as a cystic shape under an electron microscope. Exosome cargo includes a variety of biologically active substances such as non-coding RNA, lipids and small molecule proteins. Exosomes can be taken up by neighboring cells upon secretion or by distant cells within the circulatory system, affecting gene expression of the recipient cells. The present review discusses the formation and secretion of exosomes, and how they can remodel the tumor microenvironment, enhancing cancer cell chemotherapy resistance and tumor progression. Exosome-mediated induction of tumor metastasis is also highlighted. More importantly, the review discusses the manner in which exosomes can change the metabolism of cancer cells and the immune system, which may help to devise novel therapeutic approaches for cancer treatment. With the development of nanotechnology, exosomes can also be used as biomarkers and for the delivery of chemical drugs, serving as a tool to diagnose and treat cancer.
\end{abstract}

\section{Contents}

1. Introduction

2. Formation and secretion of exosomes

3. Associations between exosomes and the tumor microenvironment (TME)

4. Exosomes can enhance tumor cell chemotherapy resistance

5. Role of exosomes in tumor progression

Correspondence to: Dr Kaiyang Ding, Department of Hematology, The First Affiliated Hospital of USTC, Division of Life Sciences and Medicine, University of Science and Technology of China, 107 Huanhu Eastern Road, Hefei, Anhui 230001, P.R. China

E-mail: dingkaiy@126.com

${ }^{*}$ Contributed equally

Key words: exosomes, chemotherapy resistance, tumor metastasis, immunotherapy, biomarker, drug delivery
6. Exosomes promote tumor metastasis

7. Exosomes can alter tumor cell metabolism

8. Effects of exosomes on the immune system

9. Exosomes act as cancer biomarkers

10. Exosomes as a tumor treatment

11. Conclusion and perspectives

\section{Introduction}

In 1967, Peter Wolf first discovered that platelets can release numerous vesicles. At that time, researchers believed these vesicles were simply cell fragments with no relevant biological function (1). In 1983, exosomes were first observed in sheep reticulocytes (2). Johnstone named these vesicles 'exosomes' in 1987 and defined them as vesicles with a diameter of between 40 and $100 \mathrm{~nm}$ (3). In 2013, the Nobel Prize in physiology or medicine was awarded to James E. Rothman, Randy W. Schekman and Thomas C. Südhof for their study of extracellular vesicles (EVs) (4), which resulted in this evolving into a highly studied field.

Exosomes are EVs that originate from the endosome system and are important carriers of cell-to-cell communication in the microenvironment (5). Tumor cells can interact with immune cells, mesenchymal cells and endothelial cells in the microenvironment to promote tumor progression (6). Tumor exosomes can carry biological information in the form of proteins, lipids and nucleic acids. These molecules can domesticate recipient cells and may become tumor-specific markers (7). Therefore, exosomes and cells have significant interactions.

In clinical applications, circulating EVs derived from cancer patients are associated with tumor metastasis or recurrence; therefore, they can be used as important diagnostic and prognostic indicators, and therapeutic targets (8). In addition, exosomes, as natural nanoparticles, have great advantages over artificial materials in encapsulating chemotherapy drugs, such as paclitaxel (9). However, we do not have a complete understanding of EVs, which hinders the application of them as clinical treatments or diagnostic methods (10). Kalluri and LeBleu (11) stated that animal models with which biogenesis, trafficking and cellular entry of exosomes can be studied should be investigated rapidly. The study also summarized the challenges affecting the exosome field, such as improving the 
exosome isolation method, the influence of the administration route for exosome uptake on potential therapeutic strategies, and combining exosomal RNA, protein and metabolites to enhance the specificity and sensitivity of exosome-based diagnostics.

\section{Formation and secretion of exosomes}

Cells capture extracellular materials via endocytosis and then form early endosomes (Fig. 1). During the process of transition to a late endosome, the endocytic body sprouts inside to envelop specific proteins and nucleic acids to form intraluminal vesicles (ILVs). Advanced endosomes containing multiple ILVs are known as multivesicular bodies (MVBs) (12). Most MVBs will be digested by lysosomes in the cells. Only a few vesicles containing cluster of differentiation (CD)63, recombinant lysosomal-associated membrane protein (LAMP)1 and LAMP2 on the surface can fuse with the cell membrane and mediate the secretion of exosomes (13). ILVs and MVBs act as pre-exosomes and are formed primarily with the assistance of endosomal sorting complexes required for transport (ESCRT) protein complexes, which are mainly composed of ESCRT-0, I, II and III, as well as auxiliary proteins vacuolar protein sorting-associated protein 4 (VPS4), vacuolar protein sorting-associated protein 1 (VTA1) and apoptosis-linked gene 2 interacting protein X (ALIX). The main function of ESCRT is to sort specific substances into ILVs. ESCRT-0 contains a hepatocyte growth factor-regulated tyrosine kinase substrate (HRS) that recognizes ubiquitinated proteins and interacts with signal-transducing adaptor molecules (14). HRS recruits ESCRT-I through tumor susceptibility gene 101 protein, and then ESCRT-I recruits ESCRT-III through ESCRT-II and ALIX (15). ESCRT-III forms exosomes by cutting MVBs (16), These exosomes are then released from the cells by fusion with the cell membrane. Rabphilin (RAB)27A/B, a member of the $\mathrm{RAB}$ family, is the main motion controller of exosomes on the cytoskeleton (17) Additionally, vesicle-associated membrane protein 7 of the Soluble N-etylmaleimide-sensitive fusion protein attachment protein receptor proteins family plays the main role in promoting the fusion between exosomes and cell membranes to secrete exosomes (18). Some studies have found that Dicer and Argonaute 2, the key components of miRNA processing, are functionally present in exosomes. Although it has yet to be confirmed, these findings suggest that exosomes are not only passive vehicles but also miRNA factories $(19,20)$. Wang et al (21) reported that chemotherapeutic agents can stimulate the production or release of exosomes. Wei et al (22) found that pyruvate kinase M2, a key enzyme in aerobic glycolysis (known as the Warburg effect) of tumor cells, can promote the phosphorylation of synaptosomal-associated protein 23 and the subsequent release of exosomes. VPS33B is also a protein that regulates the release of exosomes, the deficiency of which will cause maturation and secretion disorder of exosomes (23).

\section{Associations between exosomes and the tumor microenvironment (TME)}

Exosomes were thought of as cellular metabolism waste products for a long time. With an increasing number of studies on exosomes, researchers have discovered that exosomes are important in cellular communication (24-26). Moreover, exosomes can affect tumor growth, angiogenesis, invasion and metastasis $(27,28)$. Exosomes can promote the formation of the tumor microenvironment (TME) (29), which is comprised of tumor blood vessels, the extracellular matrix (ECM) and other non-malignant cells such as stromal cells, fibroblasts and inflammatory cells $(30,31)$. Therefore, therapies that target the TME may be an effective treatment for cancer $(32,33)$. Differing from that in normal cells, the metabolism of tumor cells relies on aerobic glycolysis even under normoxic conditions. This can generate more lactic acid and thereby lower the $\mathrm{pH}$ of the TME $(34,35)$.

Exosomes can also influence the proliferation and differentiation of cells in the TME. Osteosarcoma-derived exosomes can promote osteoclast differentiation and bone resorption activity. In glioblastoma, exosomes derived from cancer cells that contain CD171 can promote glioma cell invasiveness, motility and proliferation (36). In breast cancer, MDA-MB-231 cell-derived exosomes can transfer miR-20a-5p to bone marrow macrophages and promote the proliferation and differentiation of osteoclasts by targeting SRC kinase signaling inhibitor 1 (37). Exosomes derived from mesenchymal stem cell (MSC)-differentiated adipocytes are actively taken up by breast cancer MCF7 cells. This subsequently promotes MCF7 cell proliferation and migration, as well as protecting the cells from serum deprivation or chemotherapeutic drug-induced apoptosis in vitro (38). Cancer cell-derived exosomes can promote differentiation of healthy fibroblasts or MSCs into cancer-associated fibroblasts (CAFs), which in turn activate cancer cell invasion and migration $(39,40)$. Cancer cell loss of tumor protein $\mathrm{p} 53$ can result in adrenergic trans-differentiation of tumor-associated sensory nerves by EVs (41).

\section{Exosomes can enhance tumor cell chemotherapy resistance}

Cancer cell resistance to chemotherapeutics and monoclonal antibodies is one of the difficult issues affecting the successful treatment of patients. The mechanism behind this resistance is quite complicated (Table I).

In experimental studies on exosomes, it was found that drug-resistant tumor cells can transfer their drug resistance to drug-sensitive cells through exosomes. For example, the highly tumorigenic and drug-resistant cell subpopulations in glioblastoma multiforme (GBM) can regulate other subpopulations through microRNAs (miRNAs/miRs) in EVs (42). Long non-coding (lnc)RNA regulator of Akt signaling associated with hepatocellular carcinoma and renal cell carcinoma exosomes can competitively bind miR-34/miR-449 to increase the expression of lipoxin A4 receptor and cellular-mesenchymal epithelial transition factor in RCC cells, regulating the resistance of these cells to sunitinib (43). Vemurafenib can induce expression of miR-211-5p in melanoma cells, while cells overexpressing this miRNA will deliver miR-211-5p to other melanoma cells through exosomes, and thereby enhance drug resistance (44). Gemcitabine promotes the expression of miR-155 in exosomes secreted by pancreatic ductal adenocarcinoma (PDAC) cells and can deliver it to other PDAC cells to increase their drug resistance (45). Another study reported 


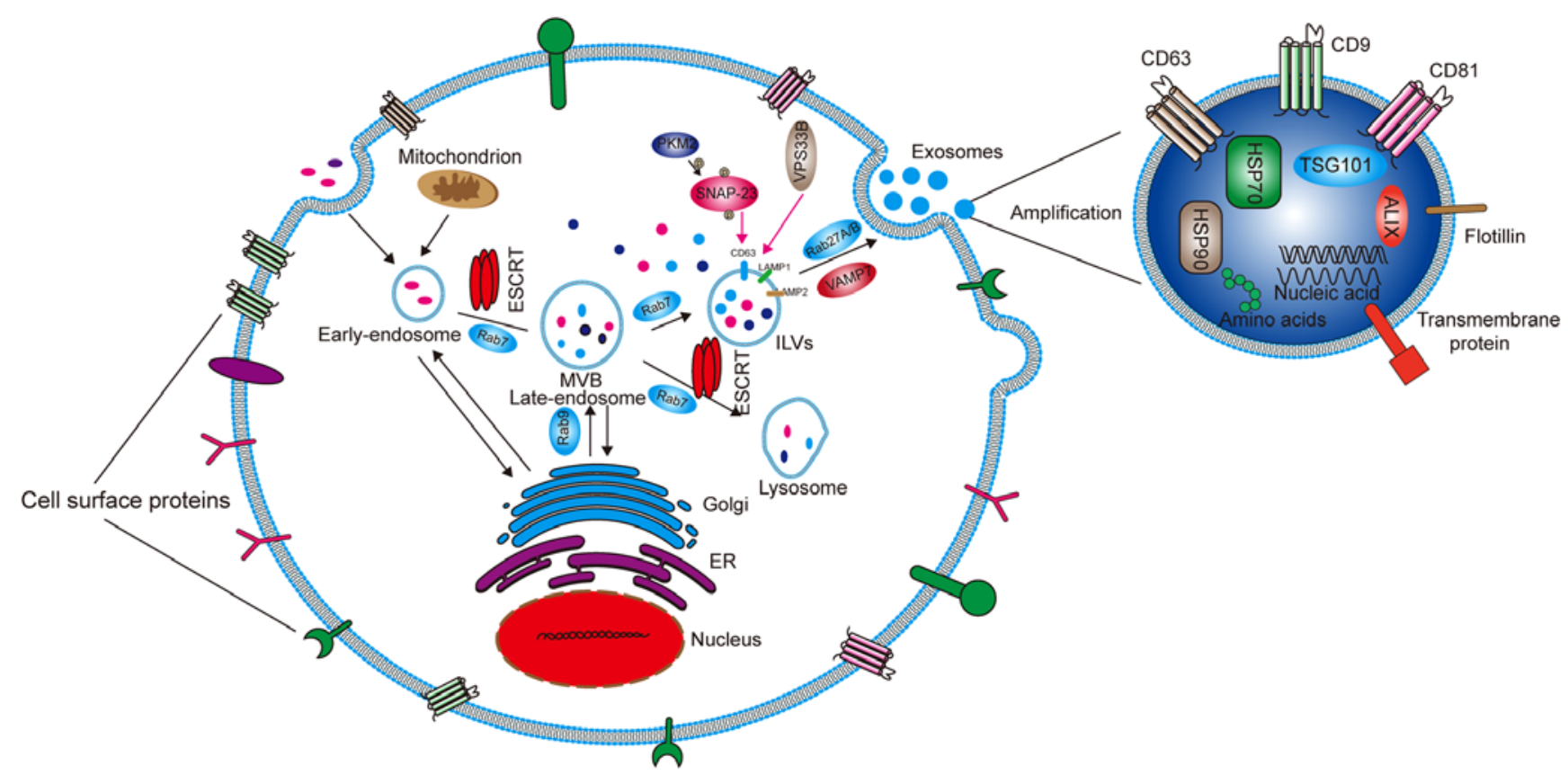

Figure 1. Formation and secretion mechanism of exosomes and the main components of exosomes. Early endosomes are formed through endocytosis, and then as a result of ESCRT, become late endosomes and then MVBs. After screening, some MVBs become ILVs and some are digested by lysosomes. ILVs are broken down to form exosomes and then released outside the cell. The whole process requires the action of Rab (an ATP protein) to provide ATP. The exosomes mainly contain HSP70, HSP90, TSG101, ALIX, amino acids and nucleic acids. The membrane surface mainly contains transmembrane proteins such as CD9, CD63, CD81 and Flotilin. ESCRT, endosomal sorting complexes required for transport; MVB, multivesicular body; ILV, intraluminal vesicle; Rab, rabphilin; HSP, heat shock protein; TSG101, tumor susceptibility gene 101 protein; ALIX, apoptosis-linked gene 2 interacting protein X; CD, cluster of differentiation; ER, endoplasmic reticulum; VAMP, vesicle-associated membrane protein; LAMP, recombinant lysosomal associated membrane protein; SNAP-23, synaptosomal-associated protein 23; VSP33B, vacuolar protein sorting-associated protein 33B; PKM2, pyruvate kinase M2.

that exosomes derived from ontogenically transformed, mesenchymal human bronchial epithelial cells (HBECs) could transfer chemoresistance to the parental, epithelial HBECs and increase zinc finger E-box binding homeobox $1 \mathrm{mRNA}$, a master epithelial-mesenchymal transition (EMT) transcription factor, in the recipient cells (46).

Moreover, exosomes secreted from stromal cells in the TME can also activate tumor-related pathways to enhance tumor cell resistance to drugs. The decreased sensitivity of breast cancer to anthracycline chemotherapy may be from exosomes secreted by peripheral cells promoting the expression of miR-125b in breast cancer cells (BCCs) (47). EVs from bone marrow stromal cells can enhance the resistance of chronic lymphocytic leukemia B cells to several chemotherapy drugs, such as fludarabine, ibrutinib, idelaisib and venetoclax (48). Exosomal miR-92a-3p derived from cancer-associated fibroblasts (CAFs) inhibits F-box/WD repeat-containing protein 7 and modulator of apoptosis 1 by activating the Wnt/ $/$-catenin pathway, and ultimately inhibits mitochondria-related apoptosis (49). In PDAC, after the first-line gemcitabine treatment, release of CAF exosomes can increase the expression of epithelial chemotherapy resistance factor Snail and promote tumor resistance and proliferation (50). Physical factors will also affect the exosomes secreted by tumor cells. In a previous study, the phosphorylation of anaplastic lymphoma kinase (ALK) in exosomes released by the non-small lung cancer H3122 cell line after irradiation was enhanced, and H3122 cells treated with these exosomes showed significant resistance to ALK-specific inhibitors such as crizotinib, ceritinib and TAE684 (51).
Finally, EV-VEGF90K derived from BCCs can interact with heat shock protein (Hsp)90, blocking the antivascular effect of bevacizumab, which can be eliminated by Hsp inhibitors (52).

\section{Role of exosomes in tumor progression}

Exosomes act as a communication tool in the TME and promote tumor growth and invasion. Firstly, the exosomes produced by stromal cells in tumors have an impact on tumor cells. For example, Lazar et al (53) found that, in the presence of adipocyte exosomes, fatty acid oxidation is increased in melanoma cells, leading to increased migration and invasion. Secondly, exosomes secreted by tumor cells can also affect stromal cells to promote tumor cell metastasis and invasion. $\alpha$-smooth muscle actin is a common biomarker of CAFs. Highly metastatic (HCC) cell-derived exosomal miR-1247-3p directly targets $\beta-1,4$-galactosyltransferase III, resulting in activation of the $\beta 1$-integrin-nuclear factor- $\kappa \mathrm{B}$ signaling pathway in fibroblasts. Activated CAFs further promote cancer progression by secreting pro-inflammatory cytokines, including interleukin (IL)-6 and IL-8 (54). Additionally, exosomal miR-105 derived from BCCs repress the expression of MAX-interacting protein 1 (an antagonist of MYC transcriptional activity) in CAFs, thereby inducing its MYC activation-related gene expression and promoting tumor cell growth (55). Notably, McAtee et al (56) found that treating prostate stromal cells with tumor exosomes robustly stimulated their migration in a manner dependent on hyaluronidases 1 (Hyal1) catalytic activity, which explains 


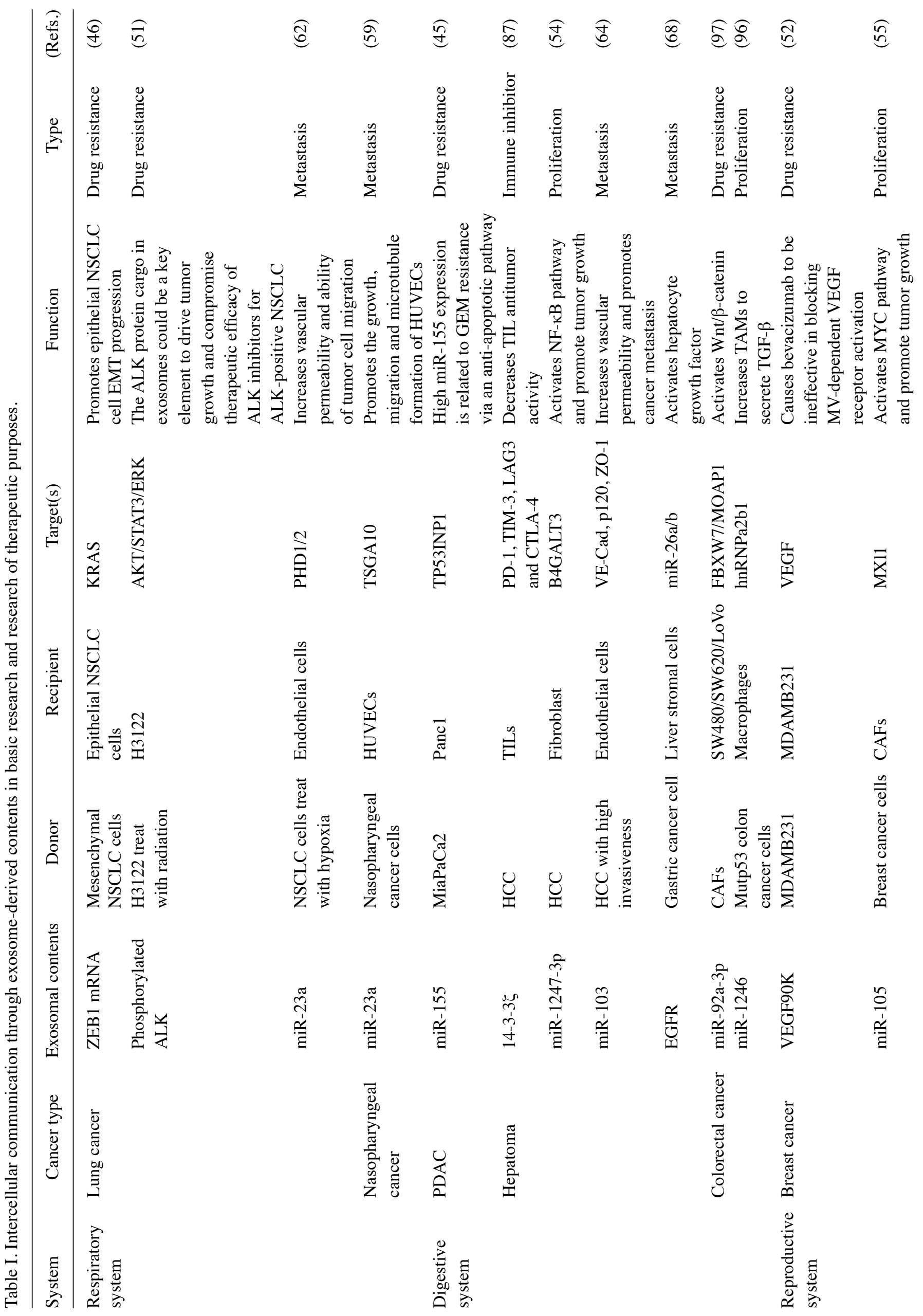




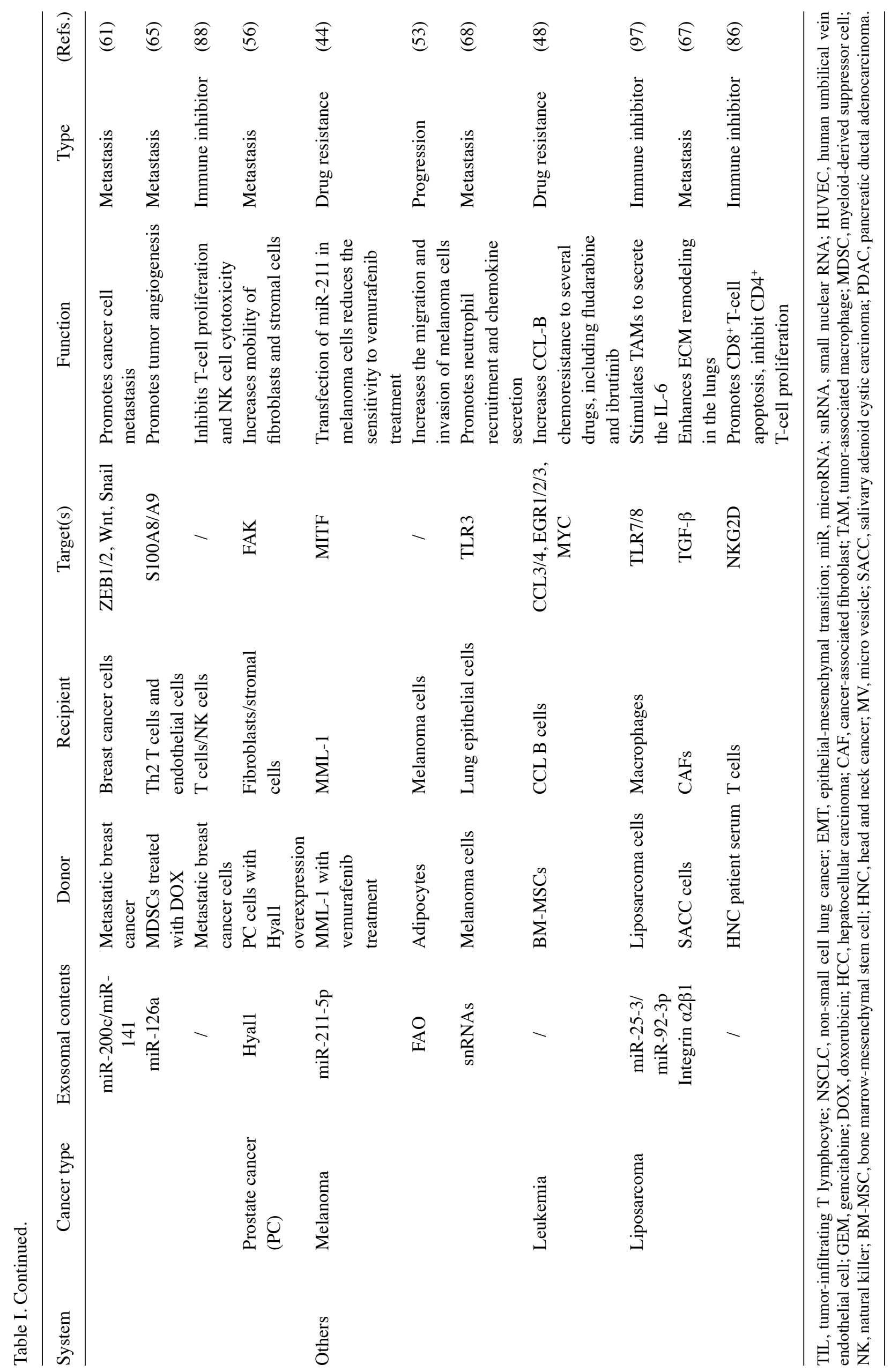




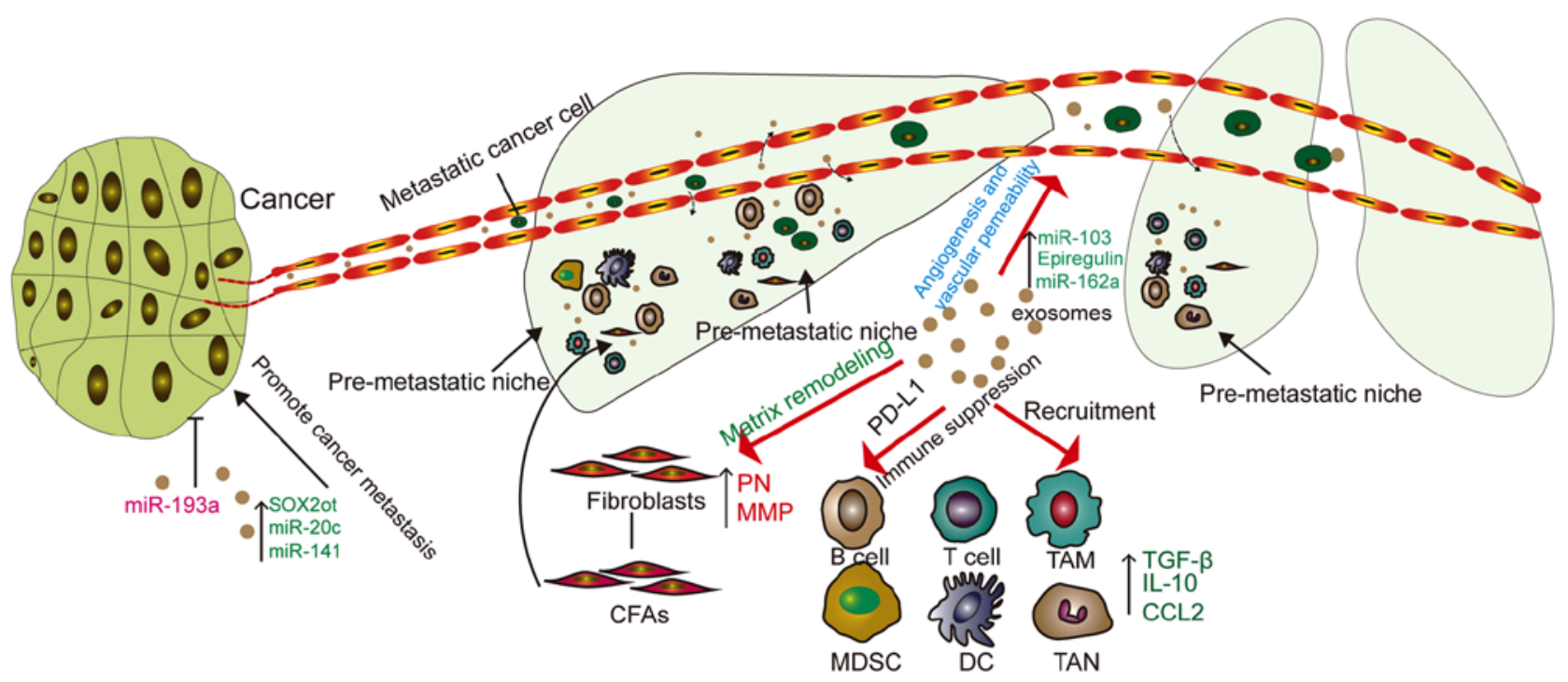

Figure 2. Functions of TDEs in cancer metastasis. TDEs can increase the permeability of blood vessels, which means that cancer cells are more likely to pass through blood vessels and seed in organs. TDEs can also recruit fibroblasts and immune cells, leading to matrix remodeling, causing upregulation of MMP and PN, forming a pre-metastatic niche and promoting tumor metastasis. TDE, tumor derived exosome; miR, microRNA; MMP, matrix metalloproteinase; SOX2ot, SRY-related high-mobility-group box protein-2 overlapping transcript; CAF, cancer-associated fibroblast; MDSC, myeloid-derived suppressor cell; DC, dendritic cell; TAM, tumor-associated macrophage; TAN, tumor associated neutrophil; CCL2, cell chemokine ligand 2 ; TGF- $\beta$, transforming growth factor $\beta$; IL, interleukin; PD-L1, programmed death ligand 1; PN, periostin.

why high levels of Hyal1 promote prostate cancer progression. In a study of the interaction between cancer cells and fibroblasts, Becker et al (57) found that tumor-derived EVs can cause differentiation of fibroblasts into myofibroblasts, which release matrix metalloproteinase (MMP) and remodel the ECM.

Exosomes secreted by tumor cells can change the characteristics of vascular endothelial cells and promote angiogenesis. For example, in the case of tumor cell hypoxia, MSC-derived EVs regulate the formation of blood vessels through various miRNAs, such as miR-126, miR-214 and miR-296 (58). miR-23a from nasopharyngeal carcinoma is rich in exosomes, which can promote the growth, migration and microtubule formation of human umbilical vein endothelial cells (59). In breast cancer, stromal fibroblasts can acquire the hallmarks of CAFs as a result of the loss of p85 $\alpha$ expression. Exosomes derived from $\mathrm{p} 85 \alpha$-deficient fibroblasts can promote cancer progression via EMT induced by the canonical Wnt pathway (60).

\section{Exosomes promote tumor metastasis}

The cancer metastasis process involves several steps; it starts with the local infiltration of cancer cells, after which they enter the circulation through the lymphatic system or blood vessels. From the circulation, cancer cells need to enter and exit from the remote organs. Exosomes are involved in all steps of this process (Fig. 2).

Exosomes can increase the EMT effect of tumor cells. For example, in a previous study, the levels of miR-200c and miR-141 in the plasma of patients with metastatic breast cancer were significantly higher than those in the healthy group. Additionally, exosomal miR-200c and miR-141 derived from metastatic breast cancer also transferred to other breast cancer cells to promote their metastasis and EMT (61).
Lung cancer cells produce more exosomes under hypoxic conditions, and miR-23a is significantly upregulated in the exosomes from these cells. This leads to hypoxia-inducible factor- $1 \alpha$ (HIF-1 $\alpha)$ accumulation in endothelial cells, which promotes the formation and permeability of blood vessels, as well as tumor migration (62). Moreover, hypoxia also increases gastric cancer (GC) exosome release and miR-301 $\alpha-3 p$ expression in a HIF-1 $\alpha$-dependent manner, which promotes GC progression and metastasis (63). The expression level of miR-103 of exosomes in highly invasive HCC cells is significantly higher compared with that in non-metastatic HCC cells. Endothelial cells treated with highly invasive HCC cell-derived exosomes also show greater permeability (64). Unexpectedly, doxorubicin induced bone marrow stem cells to secrete exosomes containing IL-13R and miR-126a in breast cancer-bearing mice, which further induced IL13 ${ }^{+} \mathrm{T}$-helper (Th) 2 cells to promote tumor angiogenesis (65).

Exosomes secreted by tumors can promote the formation of pre-metastatic niches (PMNs). EVs secreted by tumor cells form metastatic niches at remote sites of metastasis (57). The matrix environment of the niche prior to transfer is mainly composed of fibroblasts, endothelial cells and ECM. Fibroblasts not only induce inflammation and growth factors, but also express fibronectin and MMP (66). In a mouse model, CAF-derived EVs induced PMN formation in mouse lungs, increasing salivary adenoid cystic carcinoma lung metastasis (67). Exosomes in gastric cancer cells can be delivered to the liver and integrate on the plasma membrane of liver stromal cells. Exosomal epidermal growth factor receptor can inhibit the expression of miR-26a/b and activates liver cell growth factors, providing a supportive environment for gastric cancer cells (68). The study by Liu et al (69) demonstrated that lung epithelial cells are critical for initiating neutrophil recruitment and lung metastatic niche formation by sensing tumor exosomal RNAs via Toll-like receptor 3 (TLR3), providing suitable conditions for tumor metastasis. 


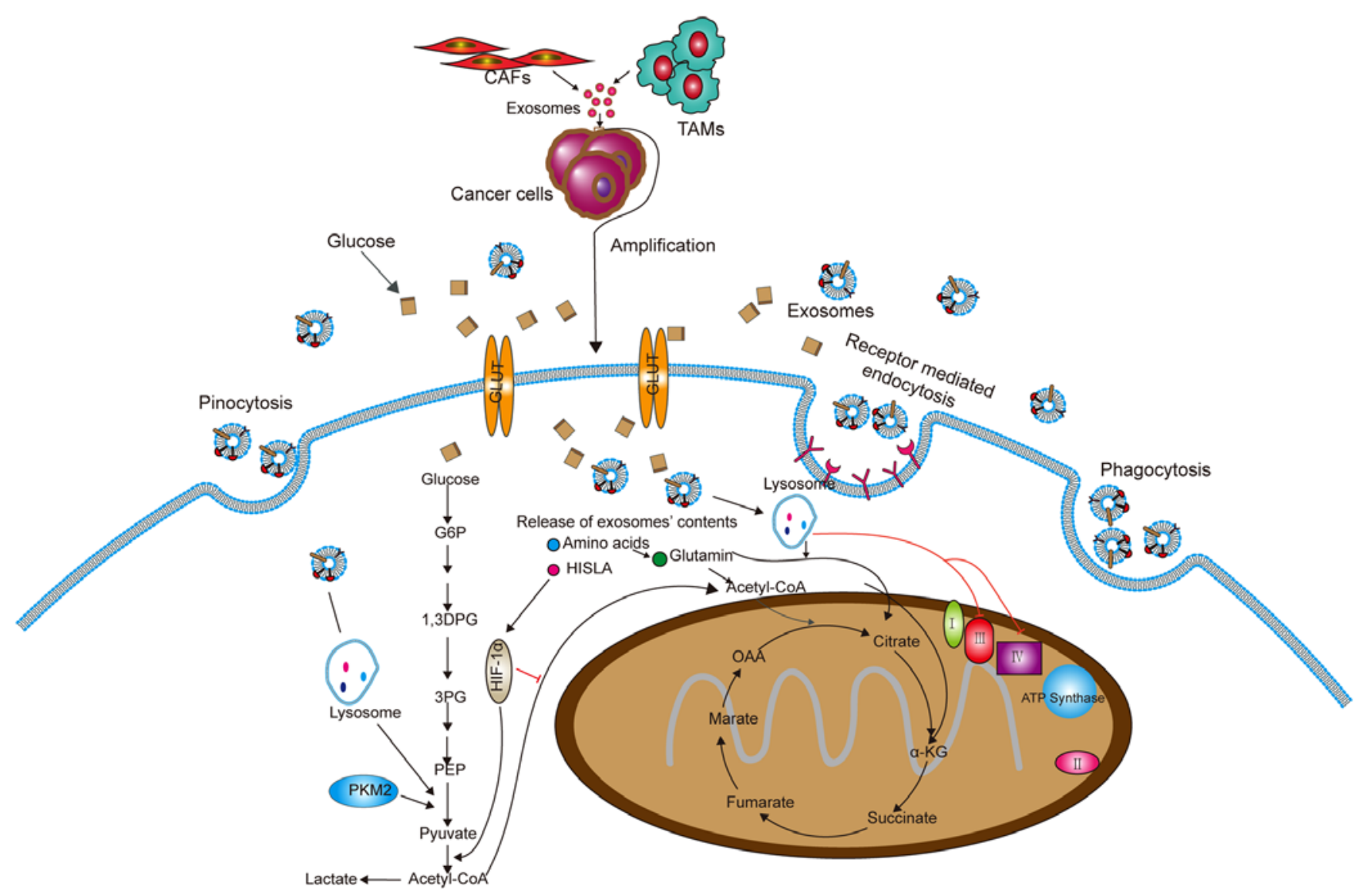

Figure 3. Exosomes derived from CAFs and TAMs can be taken up by cancer cells and change the metabolism in cancer cells. Exosomes secreted by non-tumor cells change the metabolism of tumor cells. Exosomes enter into cancer cells mainly by endocytosis, pinocytosis and receptor-mediated endocytosis. The amino acids and HISLA released from exosomes inhibit oxidative phosphorylation and cause glycolysis and glutamine-dependent reductive carboxylation. CAF, cancer-associated fibroblast; TAM, tumor-associated macrophage; HISLA, HIF-1 $\alpha$-stabilizing long non-coding RNA; PKM2, pyruvate kinase M2; PEP, phosphoenolpyruvate; 1,3DPG, 1,3-bisphosphoglyceric acid; G6P, glucose-6-phosphate; OOA, oxaloacetic acid; $\alpha$-KG, $\alpha$-ketoglutarate; GLUT, glucose transporter.

\section{Exosomes can alter tumor cell metabolism}

Metabolic reprogramming is an important characteristic of cancer cells, and this is usually caused by the TME (70). Cellular events related to the metabolic pathway include the Warburg effect, changes in Krebs cycle metabolites and the rate of oxidative phosphorylation, which may provide energy and structural requirements for the development and invasiveness of cancer cells (71) (Fig. 3). Researchers have found that exosomes secreted by CAFs in tumors can rebuild tumor metabolism. Specifically, CAF-derived exosomes could inhibit mitochondrial oxidative phosphorylation, thereby increasing glycolysis and glutamine-dependent reductive carboxylation (72). In breast cancer, the exosomes secreted by CAFs containing lncRNA small nucleolar RNA host gene 3 can sponge miR-330-5p, and miR-330-5p can target pyruvate kinase M1/M2 (PKM1/2). Therefore, exosomes from CAFs can positively regulate PKM expression, inhibit mitochondrial oxidative phosphorylation, increase glycolytic carboxylation and enhance breast tumor cell proliferation (73).

In addition, exosomes from tumor-associated macrophages (TAMs) contain HIF-1 $\alpha$-stabilizing lncRNA (HISLA). HISLA blocks the interaction of prolyl-4-hydroxylase domain-containing proteins 2 and HIF-1 $\alpha$ to inhibit the hydroxylation and degradation of HIF-1 $\alpha$, thereby promoting cancer cell oxyglycolysis (74). These findings provide a new idea for targeting exosomes to treat tumors.

\section{Effects of exosomes on the immune system}

Exosomes not only affect the growth, invasion and metastasis of tumor cells, but can also modulate the immune system of the body. Moreover, exosomes can promote anti-tumor immunity and, in a number of ways, inhibit the immune system within the TME (75) (Fig. 4).

Exosomes in the antitumor immune response. Tumor-derived exosomes or EVs can activate immune responses. According to various reports, exosomes from tumor cells present neoantigens and/or major histocompatibility complex (MHC) peptide complexes, trigger and activate $\mathrm{T}$ cells through direct presentation and cross presentation through dendritic cells (DCs), or directly activate natural killer (NK) cells or macrophages (76-78). For example, HSP70-80 and MHC-I molecules of exosomes derived from tumor antigens and tumors can interact with DCs to induce effective $\mathrm{CD}^{+}$-dependent antitumor effects on mouse tumor T cells (79). In addition, studies have reported that tumor antigen proteins can be encapsulated 


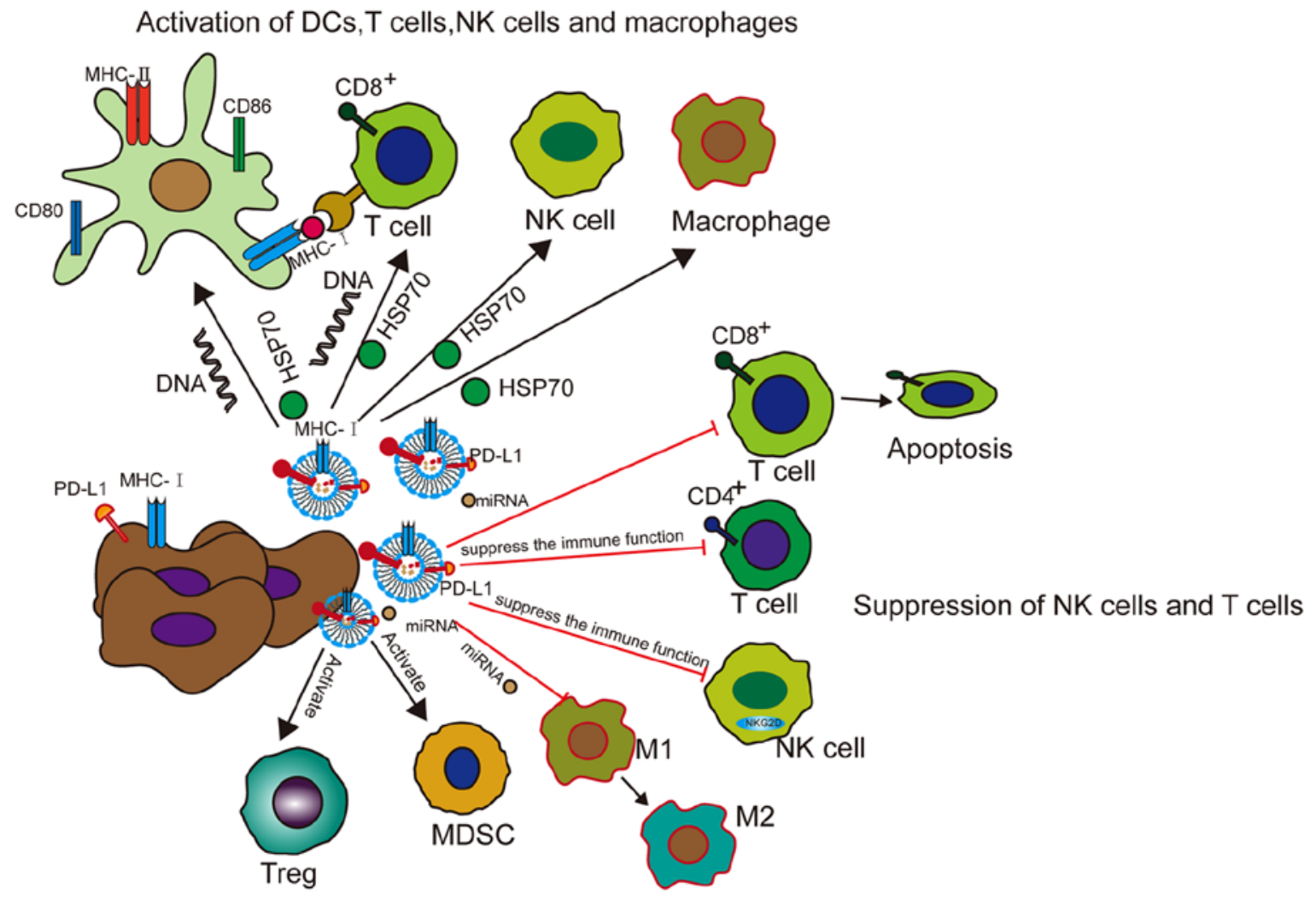

Activation of Tregs and MDSCs

Figure 4. Functions of exosomes in tumor immunity. Tumor exosomes present new antigens with MHC-1 complex (such as HSP70) to DCs or directly activate $\mathrm{T}$ cells. Tumor exosomes increase the expression of CD80, CD86 and MHC-II in DCs, thereby further activating CD4 ${ }^{+} \mathrm{T}$ cells. Exosomal DNA triggers the activation of DCs and CD8 ${ }^{+} \mathrm{T}$ cells. Tumor exosomes induce the activation of NK cells and macrophages by transferring HSP70. DCs release exosomes containing antigen and MHC-1 complex to activate cytotoxic T cells to inhibit tumor growth. To promote the tumor immune response, tumor exosomes also inhibit the functions of DCs, T cells and NK cells, enhance the population of MDSCs and Tregs, and make macrophages. The function is biased towards the M2 phenotype. Tumor exosomes carry PD-L1 from tumor cells, transfer it to DCs or macrophages, and then block T-cell function. DC, dendritic cell; MHC, major histocompatibility complex; HSP70, heat shock protein 70; CD, cluster of differentiation; NK, natural killer; MDSC, myeloid-derived suppressor cell; Treg, regulatory T cell; PD-L1, programmed death ligand 1; miRNA, microRNA.

by vesicles secreted by DCs, drained to the lymph nodes and then transferred between subpopulations of DCs. Synapses are formed between interacting DCs. Vesicle metastasis occurs in the case of exosomes. Studies have shown that DCs containing vesicles can activate $\mathrm{T}$ cells, whereas DCs lacking vesicles cannot. These results help predict the immune response and provide new methods for immunotherapy (80-82).

Treating BCCs with topotecan, an antitumor drug that causes DNA double-strand breaks, can induce the release of exosomes. Consequently, exosomal DNA derived from cancer cells treated with topotecan can trigger DC activation and subsequent $\mathrm{CD}^{+} \mathrm{T}$-cell activation through the cyclic GMP-AMP/stimulator of interferon genes signaling pathway (83).

Exosomes released from melanoma cells can be taken up by $\mathrm{CD}_{11 b^{+}}$bone marrow $(\mathrm{BM})$ cells, which can lead to the expansion and recruitment of patrolling monocytes and the differentiation of tumor necrosis factor-related apoptosis inducing ligand-positive tumor-reactive macrophages, and finally kill and phagocytose tumor cells (8).

The ability of exosomes to express antigen and MHC complexes, as well as induce helper T-cell immune responses, increases the possibility that exosomes can be used as anticancer vaccines. It is worth noting that HSP is an effective
Th1 adjuvant. Heat stress can induce the expression of HSPs and MHC-1 in tumor cells, which can result in an increase in the immunogenicity of these cells. Exosomes prepared with heat-stressed carcinoembryonic antigen (CEA)-positive tumor cells can significantly induce cytotoxic T lymphocyte (CTL) responses, indicating that exosomes derived from heat-stressed tumor cells can be used as an effective vaccine for cancer immunotherapy (84).

Tumor-supportive immune reactions. Exosomes not only play a role in antitumor immunity, but they also inhibit the immune function in tumors. Unexpectedly, exosomes participate in all known mechanisms by which cancer can evade the immune system (85).

Firstly, exosomes can modulate regulatory T cells (Tregs). For example, exosomes in the plasma of patients with head and neck cancer (HNC) carry immunosuppressive molecules and interfere with the functions of immune cells via downregulation of NK cell group 2D expression in NK cells. Exosomes in the plasma of patients with active disease significantly induce the apoptosis of $\mathrm{CD} 8^{+} \mathrm{T}$ cells, suppress $\mathrm{CD} 4^{+} \mathrm{T}$-cell proliferation and upregulate Treg suppressor functions to promote tumor growth (86). In a study on liver cancer cells, the content of $14-3-3 \zeta$ in exosomes secreted by these cells increased 
significantly. This content increased after being taken up by tumor-infiltrating $\mathrm{T}$ lymphocytes (TILs). The TILs then showed significant immune activation $\left(\mathrm{CD}^{+} 9^{+}\right)$, proliferation $\left(\mathrm{Ki}-67^{+}\right)$and decreased antitumor activity (87). Moreover, EVs can recruit and activate Tregs and myeloid-derived suppressor cells (MDSCs), and can inhibit $\mathrm{CD}^{+} \mathrm{T}$-cell-mediated apoptosis of tumor cells (56). Wen et al (88) probed exosomes secreted by mouse metastatic BCCs and found that they largely accumulated in the mouse lungs, and suppressed the immune cell functions, inhibited T-cell proliferation and decreased NK cell toxicity.

Notably, Poggio et al (89) found that exosomal programmed death ligand 1 (PD-L1) from tumor cells will suppress T-cell activation in the draining lymph node, which can promote the growth of prostate cancer cells and reduce the efficacy of immunotherapy. This also confirmed that exosomal PD-L1 can promote the formation of PMNs. Glioblastoma is a local and systemic immunosuppressive tumor that can secrete inflammatory cytokines to initiate an immune checkpoint response. In addition, EVs released by glioblastoma stem cells express PD-L1 on their surface and play a key role in mediating anti-CD3 monoclonal antibodies to inhibit $\mathrm{CD}^{+}$and $\mathrm{CD} 8^{+}$ T-cell activation and proliferation (90). In the oral/esophageal malignant lesion model induced by 4-nitroquinoline 1 oxide, PD-L1-carrying exosomes isolated from the supernatant of a mouse HNSCC cell line blocked $\mathrm{CD}^{+}{ }^{+}$and $\mathrm{CD}^{+}{ }^{+} \mathrm{T}$-cell infiltration into the TME, thereby accelerating tumor progression (91).

Haderk et al found that tumor cells can modulate the surrounding macrophages to avoid being cleared by them. For example, tumor cells can secrete exosomes, transmit immunosuppressive signals to monocytes, suppress immunity and promote tumor growth (92). Moreover, exosomes secreted by tumors can also modulate macrophages to the M2 type and promote tumor progression. For example, under hypoxic conditions, exosomes derived from epithelial ovarian cancer cells can deliver miRNAs to induce M2 macrophage polarization (93). This has also been observed in pancreatic cancer (94). In a study of head and neck cancer, the EMT transcription factor Snail directly activated miR-21 transcription in tumor cells, thereby producing miR-21-rich tumor-derived exosomes. The exosomes containing miR-21 were phagocytosed by $\mathrm{CD} 14^{+}$ monocytes, which then inhibited the expression of M1 markers and increased the expression of M2 markers (95). In colon cancer, exosomes released by cancer cells with mutated p53, are taken up by surrounding macrophages, and miR-1246 can reprogram tumor-associated macrophages (TAMs) to weaken their anti-inflammatory immune function and increase secretion of tumor growth factor (TGF)- $\beta$, thereby promoting tumor growth (96). Unexpectedly, exosomal miR-25-3p and miR-92-3p from liposarcoma stimulate TAMs to secrete the pro-inflammatory factor IL-6 in a TLR7/8-dependent manner. However, these inflammatory responses can still pass through the surrounding environment and promote the proliferation, invasion and metastasis of liposarcoma cells (97).

Overall, exosomes have both immune activation and immune suppression functions in cancer. The effects of activating immunity mainly depend on the antigen presentation of exosomes, while the immunosuppressive effects of exosomes mainly depend on the ligands, proteins and miRNAs they carry, which inhibit the activity of cytotoxic T cells or increase immunosuppressive cells. Understanding the underlying mechanisms of these two functions can help lead to the development of exosomes as a novel method to treat cancer.

\section{Exosomes act as cancer biomarkers}

Early diagnosis of tumors has always been a key factor in tumor treatment. The clinical treatment of benign tumors generally has a good prognosis. As exosomes exist in various body fluids of the human body, including the saliva, urine and blood, they can possibly be used as biomarkers for disease diagnosis and prognosis (98) (Table II).

Increased levels of circulating EVs are found in some common liver diseases such as hepatitis and liver cancer (99). In lung cancer, tumor-derived exosomal miRNAs include adenocarcinoma-specific miR-181-5p, miR-30a-3p, miR-30e-3p and miR-361-5p, as well as squamous cell carcinoma-specific miR-10b-5p, miR-15b-5p and miR-320b. These miRNAs can be used as early diagnosis markers of lung cancer (100). The expression level of IncRNA-urothelial cancer-associated 1 (IncRNA-UCA1) in the exosomes from hypoxic bladder cancer cells was significantly increased, and the expression level of lncRNA-UCA1 in the serum exosomes of patients with bladder cancer was also significantly increased. This lncRNA can therefore be used as a diagnostic biomarker for bladder cancer (101). Prostate cancer is a common cancer in men. The gold standard for diagnosis is a biopsy, which can easily cause infection and bleeding. Evaluating the recurrence by assessing the prostate-specific antigen (PSA) content in the blood is insufficient. However, examination of prostate cancer using exosomes in urine is particularly simple and fast. Moreover, the exosomes secreted by the tumor increase based on the acidic environment of this cancer, so that patients can express CD81 and PSA nanovesicles at high levels (102). In one study, after the removal of the prostate tumor, the glucuronic acid, D-ribose-5-phosphate and isobutyryl-L-carnitine contents in urine EVs from patients were all 2-26 times lower compared with those prior to surgery (103). Although the treatment success rate has reached $80-90 \%$ in acute lymphoblastic leukemia (ALL), BM biopsy still causes great pain to patients. Johnson et al (104) found that exosomes secreted by ALL cells contain high levels of CD19 compared with normal cells, which can be used to diagnose ALL. Moreover, cancer risk factors can influence the synthesis and release of exosomes. Radiation can induce the release of exosome by activating p53 transcription, which can stimulate the expression of tumor suppressor activated pathway-6 (105). In a study conducted by Wu et al (106), it was found that smoking can induce the release of EVs, $>90 \%$ of which are exosomes. When neurons are infected with Zika virus, expression of neutral sphingomyelinase-2, an important molecule that regulates the production and release of exosomes, is activated (107). Numerous cancer types usually show defects in the structure and number of centrosomes. Research reports have shown that the presence of excessive centrosomes can increase the secretion of exosomes, and that lysosomal dysfunction causes cells with extra centrosomes to secrete more exosomes in PDAC $(108,109)$.

Exosomes can also be used as a prognostic indicator of treatment. In a prospective study of patients with esophageal 


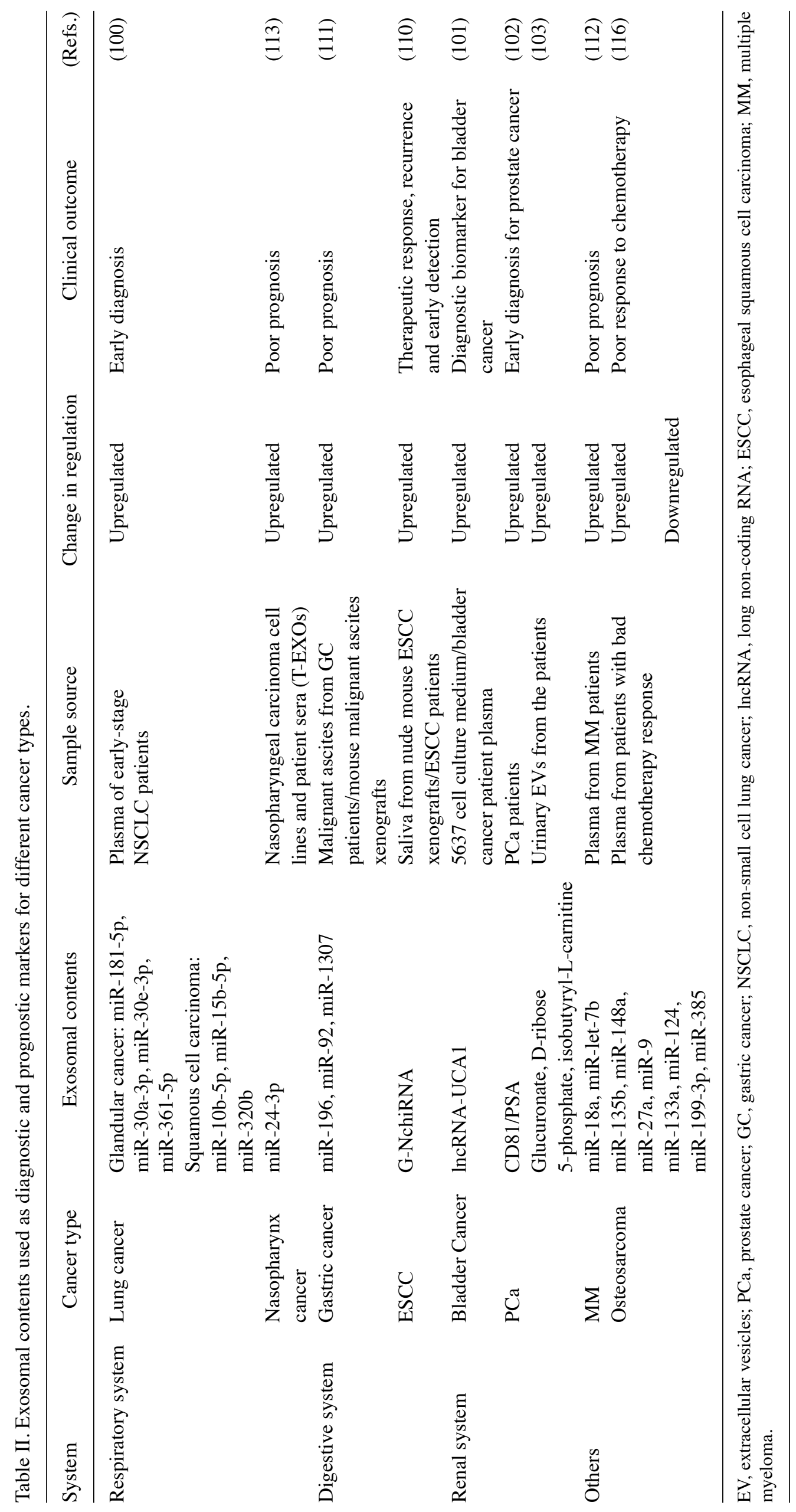



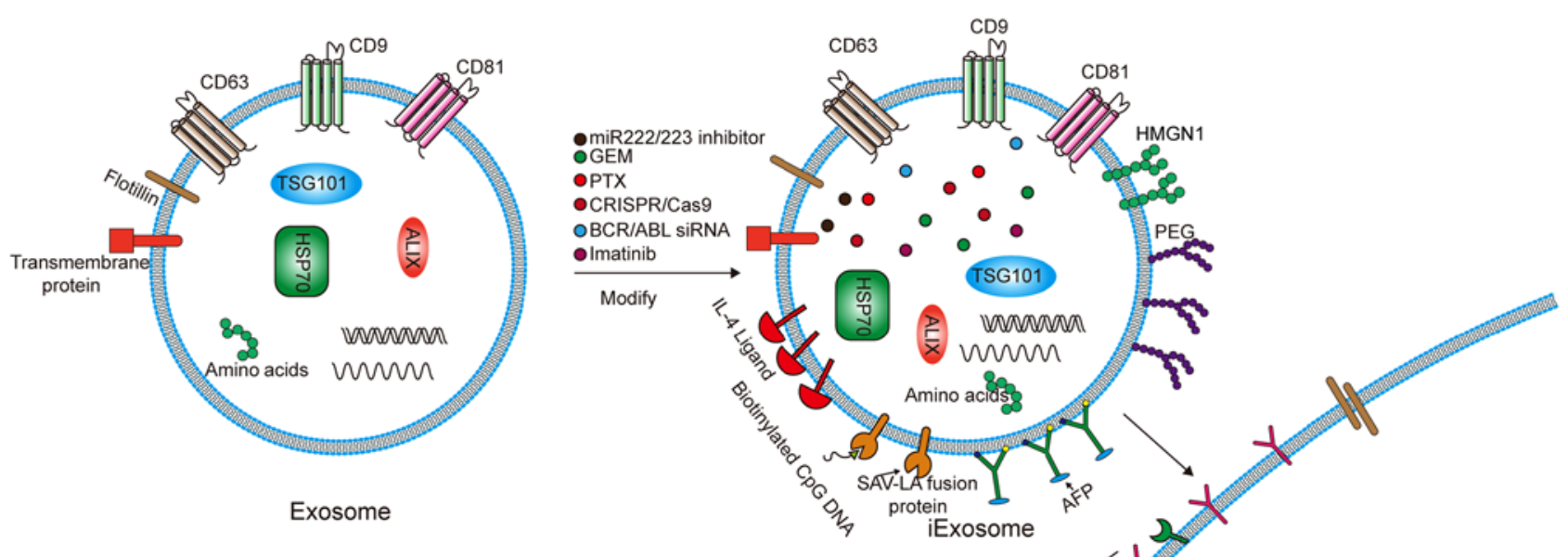

Exosome
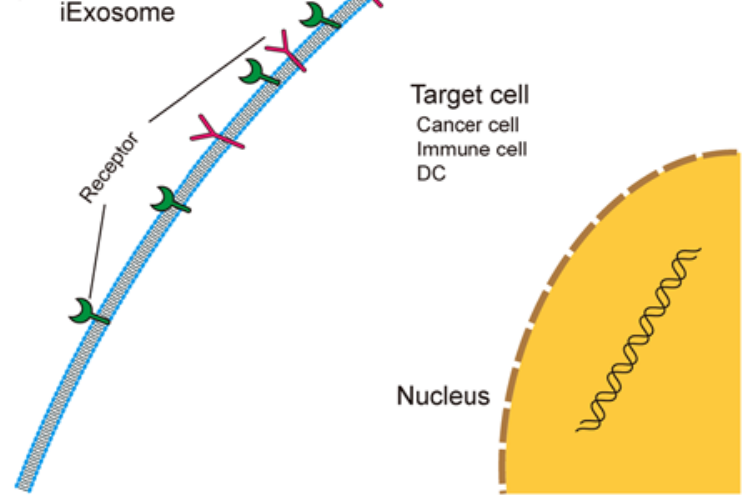

Figure 5. Exosomes can encapsulate chemical agents and be modified as engineered exosomes to deliver the agents to target cells. Exosomes can carry drugs such as GEM, PTX and imatinib and deliver them to recipient cells. Exosomes can also be loaded with the miRNA, siRNA or CRISPR/Cas9 system for tumor treatment. The surface of exosomes can be modified with SAV-LA or HMGN1 to increase the effect of antigen presentation. In addition, careful modification of the surface of exosomes with PEG or IL4 can increase the ability of the exosomes to avoid deletion and target tumor cells. GEM, gemcitabine; PTX, paclitaxel; CRISP/Cas9, clustered regularly-interspaced short palindromic repeats/CRISPR associated protein 9; BCR/ABL, breakpoint cluster region-c/abl oncogene 1; siRNA, short interfering RNA; miR, microRNA; SAV-LA, streptavidin-lactobacillus adhesion; HMGN1, high-mobility group nucleosome binding domain 1; PEG, polyethylene glycol; IL4, interleukin 4; DC, dendritic cell; HSP70, heat shock protein 70; CD, cluster of differentiation; ALIX, apoptosis-linked gene 2 interacting protein X; TSG101, tumor susceptibility gene 101 protein.

squamous cell carcinoma, the level of GOLM1-NAA35 chimeric RNA in the saliva of patients was used as a biomarker to evaluate treatment response, recurrence and early detection (110). Exosomes from the malignant ascites of patients with GC can promote the invasion of human gastric adenocarcinoma (AGS) cells. In mouse abdominal xenograft models using AGS cells, the addition of exosomes separated from malignant ascites decreased the median survival time of the mice significantly. Additionally, miR-196, miR-92 and miR-1307 are highly expressed in malignant ascites exosomes (111). Manier et al (112) showed that a cohort of 156 patients with newly diagnosed and uniformly treated multiple myeloma had exosomes containing miR-18a and miR-let-7b in the serum. Levels of these miRNAs were significantly associated with patient progression-free survival (PFS) and overall survival times. Exosomal miR-24-3p secreted by nasopharyngeal carcinoma cells can target fibroblast growth factor 11 to inhibit T-cell function. Studies have shown that patients with lower serum level of exosomal miR-24-3p have a longer PFS time (113-115). In osteosarcoma, Xu et al (116) found that in patients with OS and a poor chemotherapeutic response compared with in those with a good chemotherapeutic response, miR-133a, miR-124, miR-199-3p and miR-385 levels were significantly lower, while miR-135b, miR-148a, miR-27a and miR-9 were greatly overexpressed in serum exosomes.
These data indicate that various exosomal RNA molecules may serve as reliable biomarkers for osteosarcoma tumors with different chemotherapeutic sensitivities.

\section{Exosomes as a tumor treatment}

Exosomes, as bioactive nanovesicle substances secreted by cells, can play an important role in targeted therapy (Fig. 5). The current clinical trials of exosomes in patients with cancer are summarized in Table III. Exosomes are stable, membrane-permeable, and can even pass through the blood-brain barrier. Additionally, exosomes can be combined with a number of physical materials and can therefore be used as natural nanoparticles for drug delivery and the delivery of miRNAs/small interfering RNAs (siRNAs) to treat diseases and tumors (117).

Exosomes have a double-layer membrane structure and nanometer size, which can protect them from clearance by the cell. This prolongs their circulating half-life and improves their biological activity (118). However, the half-life of an exosome in plasma is only 2-4 min (119). A variety of strategies have been proposed to improve the tumor cell targeting specificity and tumor uptake efficiency of exosomes. For example, the engineered exosomes, iExosomes, can target the Kirsten rat sarcoma viral oncogene protein for treating 


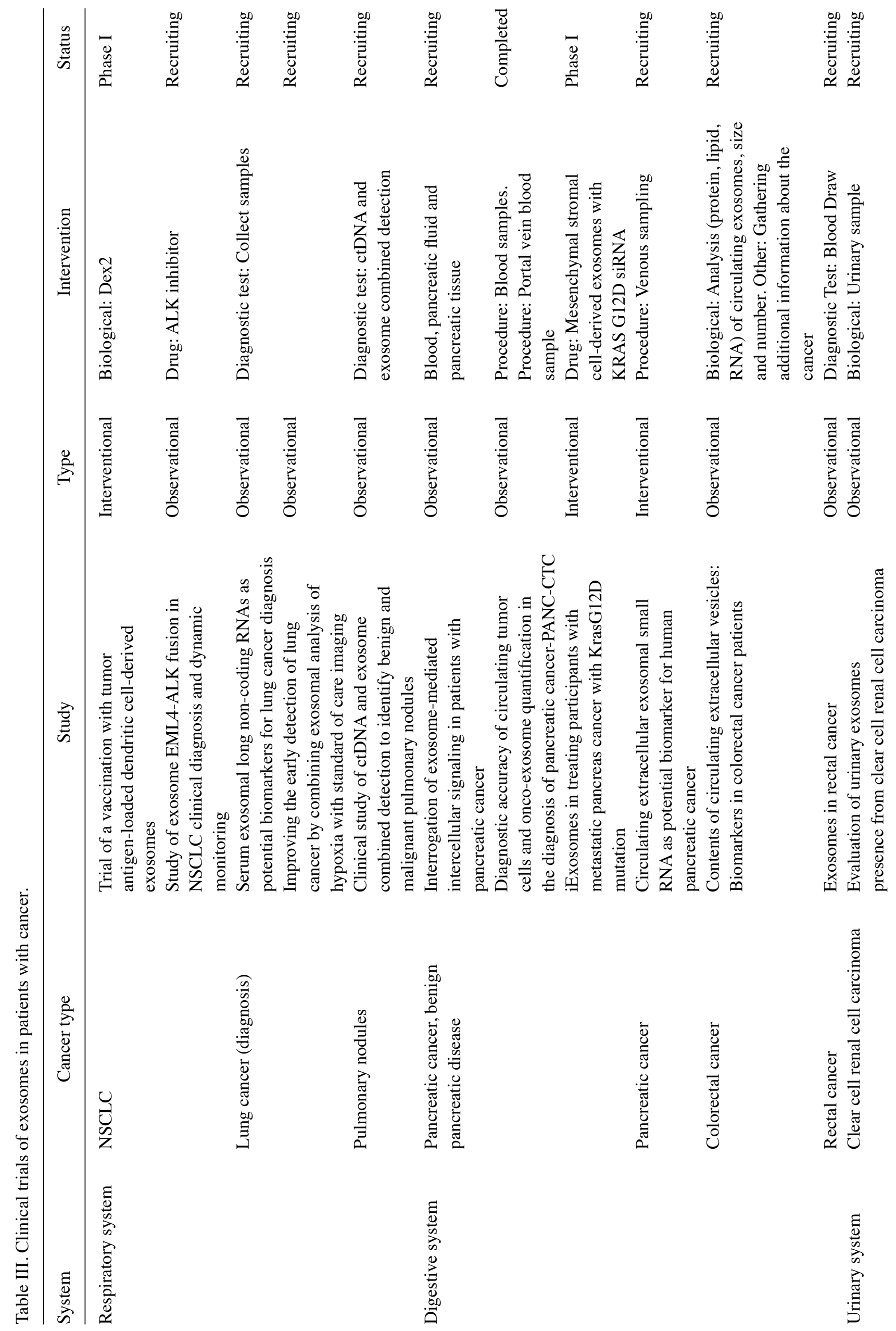




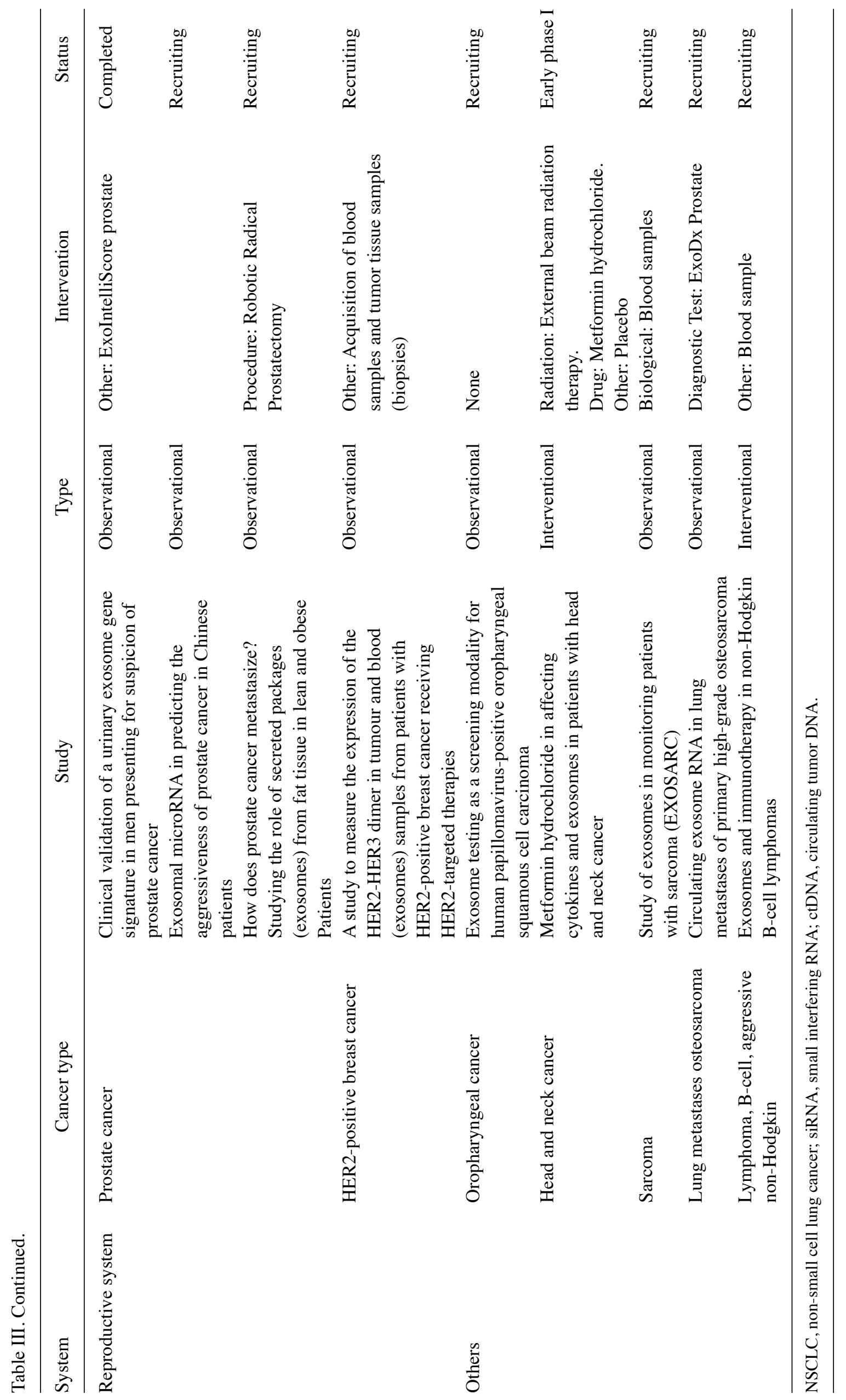


pancreatic cancer (120). In lung cancer, exosomes modified with polyethylene glycol can improve the circulation time of paclitaxel-containing exosomes in the blood. Researchers have inserted RNA nanoparticles into the membrane of exosomes with tails that carry specific ligands. This enables the exosomes to target specific tumor cells and deliver the loaded siRNA into the cells (121). Notably, exosomes can also treat drug-resistant tumor cells. Imatinib can improve the prognosis of patients with CML; however, acquired resistance is still being encountered. Bellavia et al (122) attempted to transfer imatinib or breakpoint cluster region-c-abl oncogene 1 siRNA into exosomes fused with IL-3, which targeted CML cells and inhibited tumor growth in vitro and in vivo. Bliss et al (123) reported that BCCs can prime MSCs to release exosomes containing distinct miRNA contents, such as miR-222/223, which in turn promote quiescence and confer drug resistance in a subset of cancer cells. Building on these results, a novel, nontoxic therapeutic strategy was developed to target dormant BCCs based on systemic administration of MSCs loaded with antagomiR-222/223. In an immunodeficient mouse model of dormant breast cancer, this therapy sensitized BCCs to carboplatin-based therapy and increased host survival times. Cancer-derived exosomes function as natural carriers that can efficiently deliver clustered regularly interspaced short palindromic repeats (CRISPR)/CRISPR associated protein 9 plasmids to cancer cells, inhibit the expression of poly(ADP-ribose) polymerase family member 1 and induce the death of ovarian cancer cells (124). Milk-derived exosomes have been investigated for oral delivery of the chemotherapeutic drug paclitaxel as an alternative to conventional intravenous therapy for improved efficacy and reduced toxicity (125).

Another valuable application of exosomes is their use as anticancer vaccines. Exosomes secreted by M1 macrophages can promote an inflammatory response. Encapsulating the tyrosinase-related protein 2 vaccine with lipid calcium phosphate nanoparticle enhances its activity and induces a stronger antigen-specific cytotoxic T-cell response (126). In HCC, tumor-derived exosomes (TDEs) can elicit a stronger immune response than cell lysates in vitro and in vivo (127). Exosomes derived from $\alpha$-fetoprotein (AFP)-expressing DCs (DEXAFP) and TDEs painted with the functional domain of high mobility group nucleosome-binding protein 1 elicited strong antigen-specific immune responses. Researchers found that HCC mice treated with DEXAFP had more $\gamma$-interferon (IFN- $\gamma$ )-expressing $\mathrm{CD} 8^{+} \mathrm{T}$ lymphocytes, high levels of IFN- $\gamma$ and IL-2, fewer CD25 $5^{+} \mathrm{Foxp}^{+}$Tregs, and lower levels of IL-10 and TGF $\beta$ in the tumors $(128,129)$. Japanese researchers transfected B16BL6 cells with plasmids containing streptavidin (SAV), a high-affinity knot and biotin protein, and lactobacillus adhesion (LA), an exosomal promoting protein, to produce exosomes with expression of SAV-LA. This effectively enhanced the antigen presentation capabilities of the exosomes (130).

\section{Conclusion and perspectives}

Exosomes can contain biologically active substances such as proteins, nucleic acids and lipids. Exosomes can promote cancer cell drug resistance, invasion and metastasis.
Therefore, the bioactive molecules contained within the exosomes can be used as a targeted therapy for treating tumors. In the clinic, the use of exosomes in body fluids to diagnose diseases and assess patient prognosis has also made significant progress. However, despite numerous studies and literature reports on exosomes, it remains a great challenge to translate these basic science studies to clinical applications. Most of the effects of exosomes on tumor progression or immunity have mainly been determined by 'gain-of-function' experiments, which may not fully represent the mechanism of action of exosomes in tumors. Secondly, as a biomarker for tumor diagnosis and prognosis, the extraction of exosomes is still a cumbersome task. How to extract them efficiently, rapidly and in large quantities remains a problem that needs to be addressed. Moreover, as a treatment method, the biological safety of exosomes still requires careful investigation. The next step is to decrease the cost of the extraction of exosomes, improve the safety of exosomes in clinical applications and develop a method to produce exosome-mimetic vesicles.

Although there are a number of challenges in their clinical application, exosomes are considered valid diagnostic biomarkers and potential therapeutic tools in cancer. Moreover, along with chemical, cellular and genetic engineering techniques, exosomal modification strategies may be promising for the development of clinical therapies.

\section{Acknowledgements}

Not applicable.

\section{Funding}

No funding was received.

\section{Availability of data and materials}

Not applicable.

\section{Authors' contributions}

$\mathrm{XW}$ and $\mathrm{YZ}$ contributed equally to this study. KD is the correspondence author. XW was the main contributor in writing the manuscript. YZ was the main contributor in making tables and figures. KD was the main contributor in revising the manuscript critically for important content. All authors have read and approved the manuscript. Data authentication is not applicable.

\section{Ethics approval and consent to participate}

Not applicable.

\section{Patient consent for publication}

Not applicable.

\section{Competing interests}

The authors declare that they have no competing interests. 


\section{References}

1. Wolf P: The nature and significance of platelet products in human plasma. Br J Haematol 13: 269-288, 1967.

2. Pan BT and Johnstone RM: Fate of the transferrin receptor during maturation of sheep reticulocytes in vitro: Selective externalization of the receptor. Cell 33: 967-978, 1983

3. Johnstone RM, Adam M, Hammond JR, Orr L and Turbide C: Vesicle formation during reticulocyte maturation. Association of plasma membrane activities with released vesicles (exosomes) J Biol Chem 262: 9412-9420, 1987.

4. Bonifacino JS: Vesicular transport earns a nobel. Trends Cell Biol 24: 3-5, 2014

5. Jadli AS, Ballasy N, Edalat P and Patel VB: Inside(sight) of tiny communicator: Exosome biogenesis, secretion, and uptake. Mol Cell Biochem 467: 77-94, 2020.

6. Ruivo CF, Adem B, Silva M and Melo SA: The biology of cancer exosomes: Insights and new perspectives. Cancer Res 77 6480-6488, 2017.

7. Kok VC and Yu CC: Cancer-derived exosomes: Their role in cancer biology and biomarker development. Int $\mathrm{J}$ Nanomedicine 15: 8019-8036, 2020.

8. Plebanek MP, Angeloni NL, Vinokour E, Li J, Henkin A, Martinez-Marin D, Filleur S, Bhowmick R, Henkin J, Miller SD, et al: Pre-metastatic cancer exosomes induce immune surveillance by patrolling monocytes at the metastatic niche. Nat Commun 8: 1319, 2017.

9. Kim MS, Haney MJ, Zhao Y, Mahajan V, Deygen I, Klyachko NL, Inskoe E, Piroyan A, Sokolsky M, Okolie O, et al: Development of exosome-encapsulated paclitaxel to overcome MDR in cancer cells. Nanomedicine 12: 655-664, 2016.

10. Möller A and Lobb RJ: The evolving translational potential of small extracellular vesicles in cancer. Nat Rev Cancer 20 697-709, 2020.

11. Kalluri R and LeBleu VS: The biology, function, and biomedical applications of exosomes. Science 367: eaau6977, 2020.

12. Simons M and Raposo G: Exosomes-vesicular carriers for intercellular communication. Curr Opin Cell Biol 21: 575-581, 2009.

13. Fujita Y, Kosaka N, Araya J, Kuwano K and Ochiya T: Extracellular vesicles in lung microenvironment and pathogenesis. Trends Mol Med 21: 533-542, 2015.

14. Hoshino D, Kirkbride KC, Costello K, Clark ES, Sinha S, Grega-Larson N, Tyska MJ and Weaver AM: Exosome secretion is enhanced by invadopodia and drives invasive behavior. Cell Rep 5: 1159-1168, 2013.

15. Colombo M, Raposo $\mathrm{G}$ and Thery $\mathrm{C}$ : Biogenesis, secretion, and intercellular interactions of exosomes and other extracellular vesicles. Annu Rev Cell Dev Biol 30: 255-289, 2014.

16. Frydrychowicz M, Koleckabednarczyk A, Madejczyk M, Yasar S and Dworacki G: Exosomes-structure, biogenesis and biological role in non-small-cell lung cancer. Scand J Immunol 81: 2-10, 2015.

17. Frühbeis C, Fröhlich D, Kuo WP, Amphornrat J, Thilemann S, Saab AS, Kirchhoff F, Möbius W, Goebbels S, Nave KA, et al: Neurotransmitter-triggered transfer of exosomes mediates oligodendrocyte-neuron communication. PLoS Biol 11: e1001604, 2013.

18. Zylbersztejn $\mathrm{K}$ and Galli T: Vesicular traffic in cell navigation. FEBS J 278: 4497-4505, 2011.

19. Tran N: Cancer exosomes as miRNA factories. Trends Cancer 2 : 329-331, 2016.

20. Melo SA, Sugimoto H, O'Connell JT, Kato N, Villanueva A, Vidal A, Qiu L, Vitkin E, Perelman LT, Melo CA, et al: Cancer exosomes perform cell-independent microRNA biogenesis and promote tumorigenesis. Cancer Cell 26: 707-721, 2014.

21. Wang J, Yeung BZ, Cui M, Peer CJ, Lu Z, Figg WD, Guillaume Wientjes M, Woo S and Au JL: Exosome is a mechanism of intercellular drug transfer: Application of quantitative pharmacology. J Control Release 268: 147-158, 2017.

22. Wei Y, Wang D, Jin F, Bian Z, Li L, Liang H, Li M, Shi L, Pan C, Zhu D, et al: Pyruvate kinase type M2 promotes tumour cell exosome release via phosphorylating synaptosome-associated protein 23. Nat Commun 8: 14041, 2017.

23. Gu H, Chen C, Hao X, Wang C, Zhang X, Li Z, Shao H, Zeng H, Yu Z, Xie L, et al: Sorting protein VPS33B regulates exosoma autocrine signaling to mediate hematopoiesis and leukemogenesis. J Clin Invest 126: 4537-4553, 2016.

24. Fan Q, Yang L, Zhang X, Peng X, Wei S, Su D, Zhai Z, Hua X and $\mathrm{Li} \mathrm{H}$ : The emerging role of exosome-derived non-coding RNAs in cancer biology. Cancer Lett 414: 107-115, 2018.
25. Bobrie A, Colombo M, Raposo G and Théry C: Exosome secretion: Molecular mechanisms and roles in immune responses. Traffic 12: 1659-1668, 2011

26. Li I and Nabet BY: Exosomes in the tumor microenvironment as mediators of cancer therapy resistance. Mol Cancer 18: 32, 2019

27. Wortzel I, Dror S, Kenific CM and Lyden D: Exosome-mediated metastasis: Communication from a distance. Dev Cell 49: 347-360, 2019.

28. Sung BH, Ketova T, Hoshino D, Zijlstra A and Weaver AM: Directional cell movement through tissues is controlled by exosome secretion. Nat Commun 6: 7164, 2015.

29. Hoshino A, Costa-Silva B, Shen TL, Rodrigues G, Hashimoto A, Tesic Mark M, Molina H, Kohsaka S, Di Giannatale A, Ceder S, et al: Tumour exosome integrins determine organotropic metastasis. Nature 527: 329-335, 2015.

30. Milane L, Singh A, Mattheolabakis G, Suresh M and Amiji MM: Exosome mediated communication within the tumor microenvironment. J Control Release 219: 278-294, 2015.

31. Junttila MR and de Sauvage FJ: Influence of tumour micro-environment heterogeneity on therapeutic response. Nature 501: 346-354, 2013

32. Tsukamoto H, Fujieda K, Senju S, Ikeda T, Oshiumi H and Nishimura Y: Immune-suppressive effects of interleukin-6 on T-cell-mediated anti-tumor immunity. Cancer Sci 109: 523-530, 2018.

33. Nishikawa $\mathrm{H}$ and Sakaguchi S: Regulatory $\mathrm{T}$ cells in cancer immunotherapy. Curr Opin Immunol 27: 1-7, 2014.

34. Warburg O, Wind F and Negelein E: The metabolism of tumors in the body. J Gen Physiol 8: 519-530, 1927.

35. Gatenby RA, Gawlinski ET, Gmitro AF, Kaylor BM and Gillies RJ: Acid-mediated tumor invasion: A multidisciplinary study. Cancer Res 66: 5216-5223, 2006.

36. Pace KR, Dutt R and Galileo DS: Exosomal L1CAM stimulates glioblastoma cell motility, proliferation, and invasiveness. Int J Mol Sci 20: 3982, 2019

37. Guo L, Zhu Y, Li L, Zhou S, Yin G, Yu G and Cui H: Breas cancer cell-derived exosomal miR-20a-5p promotes the proliferation and differentiation of osteoclasts by targeting SRCIN1. Cancer Med 8: 5687-5701, 2019.

38. Wang S, Su X, Xu M, Xiao X, Li X, Li H, Keating A and Zhao RC Exosomes secreted by mesenchymal stromal/stem cell-derived adipocytes promote breast cancer cell growth via activation of Hippo signaling pathway. Stem Cell Res Ther 10: 117, 2019.

39. Lee FT, Mountain AJ, Kelly MP, Hall C, Rigopoulos A, Johns TG, Smyth FE, Brechbiel MW, Nice EC, Burgess AW and Scott AM: Enhanced efficacy of radioimmunotherapy with 90Y-CHX-A'-DTPA-hu3S193 by inhibition of epidermal growth factor receptor (EGFR) signaling with EGFR tyrosine kinase inhibitor AG1478. Clin Cancer Res 11: 7080s-7086s, 2005.

40. Luo F, Sun Z, Han Q, Xue C and Bai C: Effect of human hepatocellular carcinoma HepG2 Cell-derived exosome on the differentiation of mesenchymal stem cells and their interaction. Zhongguo Yi Xue Ke Xue Yuan Xue Bao 39: 312-317, 2017.

41. Amit M, Takahashi H, Dragomir MP, Lindemann A, Gleber-Netto FO, Pickering CR, Anfossi S, Osman AA, Cai Y, Wang R, et al: Loss of p53 drives neuron reprogramming in head and neck cancer. Nature 578: 449-454, 2020.

42. Godlewski J, Ferrerluna R, Rooj AK, Mineo M, Ricklefs F, Takeda YS, Nowicki MO, Salińska E, Nakano I, Lee H, et al: MicroRNA signatures and molecular subtypes of glioblastoma: The role of extracellular transfer. Stem Cell Reports 8: 1497-1505, 2017.

43. Qu L, Ding J, Chen C, Wu ZJ, Liu B, Gao Y, Chen W, Liu F, Sun W, Li XF, et al: Exosome-transmitted lncARSR promotes sunitinib resistance in renal cancer by acting as a competing endogenous RNA. Cancer Cell 29: 653-668, 2016.

44. Lunavat TR, Cheng L, Einarsdottir BO, Olofsson Bagge R, Veppil Muralidharan S, Sharples RA, Lässer C, Gho YS, Hill AF, Nilsson JA and Lötvall J: BRAF ${ }^{\mathrm{V} 600}$ inhibition alters the microRNA cargo in the vesicular secretome of malignant melanoma cells. Proc Natl Acad Sci SA 114: E5930-E5939, 2017.

45. Mikamori M, Yamada D, Eguchi H, Hasegawa S, Kishimoto T, Tomimaru Y, Asaoka T, Noda T, Wada H, Kawamoto K, et al: MicroRNA-155 controls exosome synthesis and promotes gemcitabine resistance in pancreatic ductal adenocarcinoma. Sci Rep 7: 42339, 2017.

46. Lobb RJ, Van Amerongen R, Wiegmans AP, Ham S, Larsen JE and Möller A: Exosomes derived from mesenchymal non-small cell lung cancer cells promote chemoresistance. Int J Cancer 141: 614-620, 2017. 
47. Bach D, Hong J, Park HJ and Lee SK: The role of exosomes and miRNAs in drug-resistance of cancer cells. Int J Cancer 141: 220-230, 2017.

48. Crompot E, Van Damme M, Pieters K, Vermeersch M, Perez-Morga D, Mineur P, Maerevoet M, Meuleman N, Bron D, Lagneaux L and Stamatopoulos B: Extracellular vesicles of bone marrow stromal cells rescue chronic lymphocytic leukemia B cells from apoptosis, enhance their migration and induce gene expression modifications. Haematologica 102: 1594-1604, 2017.

49. Hu JL, Wang W, Lan X, Zeng ZC, Liang YS, Yan YR, Song FY, Wang FF, Zhu XH, Liao WJ, et al: CAFs secreted exosomes promote metastasis and chemotherapy resistance by enhancing cell stemness and epithelial-mesenchymal transition in colorectal cancer. Mol Cancer 18: 91, 2019.

50. Richards KE, Zeleniak AE, Fishel ML, Wu J, Littlepage LE and Hill R: Cancer-associated fibroblast exosomes regulate survival and proliferation of pancreatic cancer cells. Oncogene 36 : 1770-1778, 2017

51. Wu H, Zeng C, Ye Y, Liu J, Mu Z, Xie Y, Chen B, Nong Q and Wu D: Exosomes from irradiated nonsmall cell lung cancer cells reduced sensitivity of recipient cells to anaplastic lymphoma kinase inhibitors. Mol Pharm 15: 1892-1900, 2018

52. Feng Q, Zhang C, Lum D, Druso JE, Blank B, Wilson KF, Welm A, Antonyak MA and Cerione RA: A class of extracellular vesicles from breast cancer cells activates VEGF receptors and tumour angiogenesis. Nat Commun 8: 14450, 2017.

53. Lazar I, Clement E, Dauvillier S, Milhas D, Ducoux-Petit M, LeGonidec S, Moro C, Soldan V, Dalle S, Balor S, et al: Adipocyte exosomes promote melanoma aggressiveness through fatty acid oxidation: A novel mechanism linking obesity and cancer. Cancer Res 76: 4051-4057, 2016.

54. Fang T, Lv H, Lv G, Li T, Wang C, Han Q, Yu L, Su B, Guo L, Huang S, et al: Tumor-derived exosomal miR-1247-3p induces cancer-associated fibroblast activation to foster lung metastasis of liver cancer. Nat Commun 9: 191, 2018.

55. Yan W, Wu X, Zhou W, Fong MY, Cao M, Liu J, Liu X, Chen CH Fadare O, Pizzo DP, et al: Cancer-cell-secreted exosomal miR-105 promotes tumour growth through the MYC-dependent metabolic reprogramming of stromal cells. Nat Cell Biol 20 597-609, 2018.

56. McAtee CO, Booth C, Elowsky C, Zhao L, Payne J, Fangman T, Caplan S, Henry MD and Simpson MA: Prostate tumor cell exosomes containing hyaluronidase Hyal1 stimulate prostate stromal cell motility by engagement of FAK-mediated integrin signaling. Matrix Biol 78-79: 165-179, 2019.

57. Becker A, Thakur BK, Weiss JM, Kim HS, Peinado H and Lyden D: Extracellular vesicles in cancer: Cell-to-cell mediators of metastasis. Cancer Cell 30: 836-848, 2016

58. Todorova D, Simoncini S, Lacroix R, Sabatier F and Dignat-George F: Extracellular vesicles in angiogenesis. Circ Res 120: 1658-1673, 2017.

59. Bao L, You B, Shi S, Shan Y, Zhang Q, Yue H, Zhang J, Zhang W, Shi Y, Liu Y, et al: Metastasis-associated miR-23a from nasopharyngeal carcinoma-derived exosomes mediates angiogenesis by repressing a novel target gene TSGA10. Oncogene 37: 2873-2889, 2018.

60. Chen Y, Zeng C, Zhan Y, Wang H, Jiang X and Li W: Aberrant low expression of $p 85 \alpha$ in stromal fibroblasts promotes breast cancer cell metastasis through exosome-mediated paracrine Wnt10b. Oncogene 36: 4692-4705, 2017.

61. Zhang G, Zhang W, Li B, Stringer-Reasor E, Chu C, Sun L, Bae S, Chen D, Wei S, Jiao K, et al: MicroRNA-200c and microRNA-141 are regulated by a FOXP3-KAT2B axis and associated with tumor metastasis in breast cancer. Breast Cance Res 19: 73, 2017.

62. Hsu YL, Hung J, Chang W, Lin YS, Pan YC, Tsai PH, Wu CY and Kuo PL: Hypoxic lung cancer-secreted exosomal miR-23a increased angiogenesis and vascular permeability by targeting prolyl hydroxylase and tight junction protein ZO-1. Oncogene 36 : 4929-4942, 2017.

63. Xia X, Wang S, Ni B, Xing S, Cao H, Zhang Z, Yu F, Zhao E and Zhao G: Hypoxic gastric cancer-derived exosomes promote progression and metastasis via MiR-301a-3p/PHD3/ HIF-1 $\alpha$ positive feedback loop. Oncogene 39: 6231-6244, 2020.

64. Fang JH, Zhang ZJ, Shang LR, Luo YW, Lin YF, Yuan Y and Zhuang SM: Hepatoma cell-secreted exosomal microRNA-103 increases vascular permeability and promotes metastasis by targeting junction proteins. Hepatology 68: 1459-1475, 2018.
65. Matei I, Kim HS and Lyden D: Unshielding exosomal RNA unleashes tumor growth and metastasis. Cell 170: 223-225, 2017.

66. Yamamura Y, Asai N, Enomoto A, Kato T, Mii S, Kondo Y, Ushida K, Niimi K, Tsunoda N, Nagino M, et al: Akt-Girdin signaling in cancer-associated fibroblasts contributes to tumor progression. Cancer Res 75: 813-823, 2015.

67. Kong J, Tian H, Zhang F, Zhang Z, Li J, Liu X, Li X, Liu J, Li X, Jin D, et al: Extracellular vesicles of carcinoma-associated fibroblasts creates a pre-metastatic niche in the lung through activating fibroblasts. Mol Cancer 18: 175, 2019.

68. Zhang H, Deng T, Liu R, Bai M, Zhou L, Wang X, Li S, Wang X, Yang H, Li J, et al: Exosome-delivered EGFR regulates liver microenvironment to promote gastric cancer liver metastasis. Nat Commun 8: 15016,2017.

69. Liu Y, Gu Y, Han Y, Zhang Q, Jiang Z, Zhang X, Huang B, $\mathrm{Xu} \mathrm{X}$, Zheng $\mathrm{J}$ and Cao $\mathrm{X}$ : Tumor exosomal RNAs promote lung pre-metastatic niche formation by activating alveolar epithelial TLR3 to recruit neutrophils. Cancer Cell 30: 243-256, 2016

70. Lane AN, Higashi RM and Fan TW: Metabolic reprogramming in tumors: Contributions of the tumor microenvironment. Genes Dis 7: 185-198, 2019.

71. Ji K, Mayernik L, Moin K and Sloane BF: Acidosis and proteolysis in the tumor microenvironment. Cancer Metastasis Rev 38: 103-112, 2019.

72. Zhao H, Yang L, Baddour J, Achreja A, Bernard V, Moss T, Marini JC, Tudawe T, Seviour EG, San Lucas FA, et al: Tumor microenvironment derived exosomes pleiotropically modulate cancer cell metabolism. Elife 5: e10250, 2016.

73. Li Y,Zhao Z, Liu W and Li X: SNHG3 functions as miRNA sponge to promote breast cancer cells growth through the metabolic reprogramming. Appl Biochem Biotechnol 191: 1084-1099, 2020.

74. Tomasetti M, Lee W, Santarelli L and Neuzil J: Exosome-derived microRNAs in cancer metabolism: Possible implications in cancer diagnostics and therapy. Exp Mol Med 49: e285, 2017

75. Greening DW, Gopal SK, Xu R, Simpson RJ and Chen W: Exosomes and their roles in immune regulation and cancer. Semin Cell Dev Biol 40: 72-81, 2015.

76. Filipazzi P, Bürdek M, Villa A, Rivoltini L and Huber V: Recent advances on the role of tumor exosomes in immunosuppression and disease progression. Semin Cancer Biol 22: 342-349, 2012.

77. Wolfers J, Lozier A, Raposo G, Regnault A, Théry C, Masurier C, Flament C, Pouzieux S, Faure F, Tursz T, et al: Tumor-derived exosomes are a source of shared tumor rejection antigens for CTL cross-priming. Nat Med 7: 297-303, 2001.

78. Dai S, Zhou X, Wang B, Wang Q, Fu Y, Chen T, Wan T, Yu Y and Cao X: Enhanced induction of dendritic cell maturation and HLA-A*0201-restricted CEA-specific CD8(+) CTL response by exosomes derived from IL-18 gene-modified CEA-positive tumor cells. J Mol Med (Berl) 84: 1067-1076, 2006.

79. Altieri SL, Khan AN and Tomasi TB: Exosomes from plasmacytoma cells as a tumor vaccine. J Immunother 27: 282-288, 2004.

80. Balan S and Bhardwaj N: Cross-presentation of tumor antigens is ruled by synaptic transfer of vesicles among dendritic cell subsets. Cancer Cell 37: 751-753, 2020.

81. Zitvogel L, Regnault A, Lozier A, Wolfers J, Flament C, Tenza D, Ricciardi-Castagnoli P, Raposo G and Amigorena S: Eradication of established murine tumors using a novel cell-free vaccine: Dendritic cell-derived exosomes. Nat Med 4: 594-600, 1998.

82. Pitt JM, André F, Amigorena S, Soria JC, Eggermont A, Kroemer G and Zitvogel L: Dendritic cell-derived exosomes for cancer therapy. J Clin Invest 126: 1224-1232, 2016.

83. Kitai Y, Kawasaki T, Sueyoshi T, Kobiyama K, Ishii KJ, Zou J, Akira S, Matsuda T and Kawai T: DNA-containing exosomes derived from cancer cells treated with topotecan activate a STING-dependent pathway and reinforce antitumor immunity. J Immunol 198: 1649-1659, 2017.

84. Dai S, Wan T, Wang B, Zhou X, Xiu F, Chen T, Wu Y and Cao X: More efficient induction of HLA-A*0201-restricted and carcinoembryonic antigen (CEA)-specific CTL response by immunization with exosomes prepared from heat-stressed CEA-positive tumor cells. Clin Cancer Res 11: 7554-7563, 2005.

85. Czernek L and Düchler M: Functions of cancer-derived extracellular vesicles in immunosuppression. Arch Immunol Ther Exp (Warsz) 65: 311-323, 2017

86. Ludwig S, Floros T, Theodoraki MN, Hong CS, Jackson EK, Lang S and Whiteside TL: Suppression of lymphocyte functions by plasma exosomes correlates with disease activity in patients with head and neck cancer. Clin Cancer Res 23: 4843-4854, 2017. 
87. Wang X, Shen H, Zhangyuan G, Huang R, Zhang W, He Q, Jin K, Zhuo H, Zhang Z, Wang J, et al: 14-3-3 $\}$ delivered by hepatocellular carcinoma-derived exosomes impaired anti-tumor function of tumor-infiltrating T lymphocytes. Cell Death Dis 9: 159, 2018.

88. Wen SW, Sceneay J, Lima LG, Wong CS, Becker M, Krumeich S, Lobb RJ, Castillo V, Wong KN, Ellis S, et al: The biodistribution and immune suppressive effects of breast cancer-derived exosomes. Cancer Res 76: 6816-6827, 2016.

89. Poggio M, Hu T, Pai CC, Chu B, Belair CD, Chang A Montabana E, Lang UE, Fu Q, Fong L and Blelloch R: Suppression of exosomal PD-L1 induces systemic anti-tumor immunity and memory. Cell 177: 414-427.e13, 2019.

90. Gabrusiewicz K, Li X, Wei J, Hashimoto Y, Marisetty AL Ott M, Wang F, Hawke D, Yu J, Healy LM, et al: Glioblastoma stem cell-derived exosomes induce M2 macrophages and PD-L1 expression on human monocytes. Oncoimmunology 7 : e1412909, 2018.

91. Razzo BM, Ludwig N, Hong CS, Sharma P, Fabian KP, Fecek RJ, Storkus WJ and Whiteside TL: Tumor-derived exosomes promote carcinogenesis of murine oral squamous cell carcinoma. Carcinogenesis 41: 625-633, 2020.

92. Haderk F, Schulz R, Iskar M, Cid LL, Worst T, Willmund KV, Schulz A, Warnken U, Seiler J, Benner A, et al: Tumor-derived exosomes modulate PD-L1 expression in monocytes. Sci Immunol 2: eaah5509, 2017

93. Chen X, Zhou J, Li X, Wang X, Lin Y and Wang X: Exosomes derived from hypoxic epithelial ovarian cancer cells deliver microRNAs to macrophages and elicit a tumor-promoted phenotype. Cancer Lett 435: 80-91, 2018.

94. Wang X, Luo G, Zhang K, Cao J, Huang C, Jiang T, Liu B, Su L and Qiu Z: Hypoxic tumor-derived exosomal miR-301a mediates M2 macrophage polarization via PTEN/PI3K $\gamma$ to promote pancreatic cancer metastasis. Cancer Res 78 4586-4598, 2018

95. Hsieh CH, Tai SK and Yang MH: Snail-overexpressing cancer cells promote M2-like polarization of tumor-associated macrophages by delivering MiR-21-abundant exosomes. Neoplasia 20: 775-788, 2018

96. Cooks T, Pateras IS, Jenkins LM, Patel KM, Robles AI, Morris J, Forshew T, Appella E, Gorgoulis VG and Harris CC: Mutant p53 cancers reprogram macrophages to tumor supporting macrophages via exosomal miR-1246. Nat Commun 9: 771, 2018.

97. Casadei L, Calore F, Creighton CJ, Guescini M, Batte K, Iwenofu OH, Zewdu A, Braggio DA, Bill KL, Fadda P, et al: Exosome-derived miR-25-3p and miR-92a-3p stimulate liposarcoma progression. Cancer Res 77: 3846-3856, 2017.

98. Whiteside TL: Tumor-derived exosomes and their role in cancer progression. Adv Clin Chem 74: 103-141, 2016.

99. Szabo G and Momen-Heravi F: Extracellular vesicles in liver disease and potential as biomarkers and therapeutic targets. Nat Rev Gastroenterol Hepatol 14: 455-466, 2017.

100. Jin X, Chen Y, Chen H, Fei S, Chen D, Cai X, Liu L, Lin B, Su H, Zhao L, et al: Evaluation of tumor-derived exosomal miRNA as potential diagnostic biomarkers for early-stage non-small cell lung cancer using next-generation sequencing. Clin Cancer Res 23: 5311-5319, 2017.

101. Xue M, Chen W, Xiang A, Wang R, Chen H, Pan J, Pang H, An $\mathrm{H}$, Wang $\mathrm{X}$, Hou $\mathrm{H}$ and $\mathrm{Li} \mathrm{X}$ : Hypoxic exosomes facilitate bladder tumor growth and development through transferring long non-coding RNA-UCA1. Mol Cancer 16: 143, 2017.

102.Logozzi M, Angelini DF, Iessi E, Mizzoni D, Di Raimo R, Federici C, Lugini L, Borsellino G, Gentilucci A, Pierella F, et al: Increased PSA expression on prostate cancer exosomes in in vitro condition and in cancer patients. Cancer Lett 403 : 318-329, 2017

103. Puhka M, Takatalo M, Nordberg ME, Valkonen S, Nandania J, Aatonen M, Yliperttula M, Laitinen S, Velagapudi V, Mirtti T, et al: Metabolomic profiling of extracellular vesicles and alternative normalization methods reveal enriched metabolites and strategies to study prostate cancer-related changes. Theranostics 7: 3824-3841, 2017.

104. Johnson SM, Dempsey C, Chadwick AL, Harrison S, Liu J, Di Y, McGinn OJ, Fiorillo M, Sotgia F, Lisanti MP, et al: Metabolic reprogramming of bone marrow stromal cells by leukemic extracellular vesicles in acute lymphoblastic leukemia. Blood 128: 453-456, 2016.

105. Yu X, Harris SL and Levine AJ: The regulation of exosome secretion: A novel function of the p53 protein. Cancer Res 66 : 4795-4801, 2006
106. Wu F, Yin Z, Yang L, Fan J, Xu J, Jin Y, Yu J, Zhang D and Yang G: Smoking induced extracellular vesicles release and their distinct properties in non-small cell lung cancer. J Cancer 10: 3435-3443, 2019.

107. Zhou W, Woodson M, Sherman MB, Neelakanta G and Sultana H: Exosomes mediate Zika virus transmission through SMPD3 neutral sphingomyelinase in cortical neurons. Emerg Microbes Infect 8: 307-326, 2019.

108. Baumann K: Making more exosomes. Nat Rev Mol Cell Biol 22: 242,2021

109. Adams SD, Csere J, D'Angelo G, Carter EP, Romao M, Arnandis T, Dodel M, Kocher HM, Grose R, Raposo G, et al: Centrosome amplification mediates small extracellular vesicle secretion via lysosome disruption. Curr Biol 31: 1403-1416.e7, 2021

110. Lin Y, Dong H, Deng W, Lin W, Li K, Xiong X, Guo Y, Zhou F, Ma C, Chen Y, et al: Evaluation of salivary exosomal chimeric GOLM1-NAA35 RNA as a potential biomarker in esophageal carcinoma. Clin Cancer Res 25: 3035-3045, 2019.

111. Hu Y, Qi C, Liu X, Zhang C, Gao J, Wu Y, Yang J, Zhao Q, Li J, Wang X and Shen L: Malignant ascites-derived exosomes promote peritoneal tumor cell dissemination and reveal a distinct miRNA signature in advanced gastric cancer. Cancer Lett 457: 142-150, 2019

112. Manier S, Liu CJ, Avet-Loiseau H, Park J, Shi J, Campigotto F, Salem KZ, Huynh D, Glavey SV, Rivotto B, et al: Prognostic role of circulating exosomal miRNAs in multiple myeloma. Blood 129: 2429-2436, 2017.

113. Ye SB, Zhang H, Cai TT, Liu YN, Ni JJ, He J, Peng JY, Chen QY, Mo HY, Jun Cui, et al: Exosomal miR-24-3p impedes T-cell function by targeting FGF11 and serves as a potential prognostic biomarker for nasopharyngeal carcinoma. J Pathol 240: 329-340, 2016

114. He L, Ping F, Fan Z, Zhang C, Deng M, Cheng B and Xia J: Salivary exosomal miR-24-3p serves as a potential detective biomarker for oral squamous cell carcinoma screening. Biomed Pharmacother 121: 109553, 2020.

115. Zou X, Zhu D, Zhang H, Zhang S, Zhou X, He X, Zhu J and Zhu W: MicroRNA expression profiling analysis in serum for nasopharyngeal carcinoma diagnosis. Gene 727: 144243, 2020.

116. Xu JF, Wang YP, Zhang SJ, Chen Y, Gu HF, Dou XF, Xia B, Bi Q and Fan SW: Exosomes containing differential expression of microRNA and mRNA in osteosarcoma that can predict response to chemotherapy. Oncotarget 8: 75968-75978, 2017.

117. Wang J, Li W, Zhang L, Ban L, Chen P, Du W, Feng X and Liu BF: Chemically edited exosomes with dual ligand purified by microfluidic device for active targeted drug delivery to tumor cells. ACS Appl Mater Interfaces 9: 27441-27452, 2017

118. He C,Zheng S, Luo Y and Wang B: Exosome theranostics: Biology and translational medicine. Theranostics 8: 237-255, 2018.

119. Charoenviriyakul C, Takahashi Y, Morishita M, Matsumoto A, Nishikawa $\mathrm{M}$ and Takakura Y: Cell type-specific and common characteristics of exosomes derived from mouse cell lines: Yield, physicochemical properties, and pharmacokinetics. Eur J Pharm Sci 96: 316-322, 2017

120. Kamerkar S, LeBleu VS, Sugimoto H, Yang S, Ruivo CF Melo SA, Lee JJ and Kalluri R: Exosomes facilitate therapeutic targeting of oncogenic KRAS in pancreatic cancer. Nature 546: 498-503, 2017.

121. Pi F, Binzel DW, Lee TJ, Li Z, Sun M, Rychahou P, Li H, Haque F, Wang S, Croce CM, et al: Nanoparticle orientation to control RNA loading and ligand display on extracellular vesicles for cancer regression. Nat Nanotechnol 13: 82-89, 2018

122. Bellavia D, Raimondo S, Calabrese G, Forte S, Cristaldi M, Patinella A, Memeo L, Manno M, Raccosta S, Diana P, et al: Interleukin 3-receptor targeted exosomes inhibit in vitro and in vivo chronic myelogenous leukemia cell growth. Theranostics 7: 1333-1345, 2017

123. Bliss SA, Sinha G, Sandiford OA, Williams LM, Engelberth DJ, Guiro K, Isenalumhe LL, Greco SJ, Ayer S, Bryan M, et al: Mesenchymal stem cell-derived exosomes stimulate cycling quiescence and early breast cancer dormancy in bone marrow. Cancer Res 76: 5832-5844, 2016.

124. Kim SM, Yang Y, Oh SJ, Hong Y, Seo M and Jang M: Cancer-derived exosomes as a delivery platform of CRISPR/Cas9 confer cancer cell tropism-dependent targeting. J Control Release 266: 8-16, 2017.

125. Agrawal AK, Aqil F, Jeyabalan J, Spencer WA, Beck J, Gachuki BW, Alhakeem SS, Oben K, Munagala R, Bondada S and Gupta RC: Milk-derived exosomes for oral delivery of paclitaxel. Nanomedicine 13: 1627-1636, 2017. 
126. Cheng L, Wang Y and Huang L: Exosomes from M1-Polarized macrophages potentiate the cancer vaccine by creating a pro-inflammatory microenvironment in the lymph node. Mol Ther 25: 1665-1675, 2017.

127. Rao Q, Zuo B, Lu Z, Gao X, You A, Wu C, Du Z and Yin H: Tumor-derived exosomes elicit tumor suppression in murine hepatocellular carcinoma models and humans in vitro. Hepatology 64: 456-472, 2016.

128. Zuo B, Qi H, Lu Z, Chen L, Sun B, Yang R, Zhang Y, Liu Z, Gao X, You A, et al: Alarmin-painted exosomes elicit persistent antitumor immunity in large established tumors in mice. Nat Commun 11: 1790, 2020.
129. Lu Z, Zuo B, Jing R, Gao X, Rao Q, Liu Z, Qi H, Guo H and Yin H: Dendritic cell-derived exosomes elicit tumor regression in autochthonous hepatocellular carcinoma mouse models. J Hepatol 67: 739-748, 2017.

130. Morishita M, Takahashi Y, Matsumoto A, Nishikawa M and Takakura Y: Exosome-based tumor antigens-adjuvant co-delivery utilizing genetically engineered tumor cell-derived exosomes with immunostimulatory CpG DNA. Biomaterials 111: 55-65, 2016.

This work is licensed under a Creative Commons Attribution-NonCommercial-NoDerivatives 4.0 International (CC BY-NC-ND 4.0) License. 